\title{
Heterogeneous Mixture Distributions for Modeling Multisource Extreme Rainfalls*
}

\author{
JU-YOUNG SHIN \\ Institute Center for Water Advanced Technology and Environmental Research, Masdar Institute of Science \\ and Technology, Abu Dhabi, United Arab Emirates \\ TAESAM LEE \\ Department of Civil Engineering, ERI, Gyeongsang National University, Jinju, South Korea \\ TAHA B. M. J. OUARDA \\ Institute Center for Water Advanced Technology and Environmental Research, Masdar Institute of Science \\ and Technology, Abu Dhabi, United Arab Emirates
}

(Manuscript received 2 July 2014, in final form 18 June 2015)

\begin{abstract}
Frequency analysis has been widely applied to investigate the behavior and characteristics of hydrometeorological variables. Hydrometeorological variables occasionally show mixture distributions when multiple generating phenomena cause the extreme events to occur. In such cases, a mixture distribution should be applied. Past studies on mixture distributions assumed that they are drawn from the same probability density functions. In fact, many hydrometeorological variables can consist of different types of probability density functions. Research on heterogeneous mixture distributions can lead to improvements in understanding the behavior and characteristics of hydrometeorological variables and in the capacity to model them properly. In the present study heterogeneous mixture distributions are developed to model extreme hydrometeorological events. To fit heterogeneous mixture distributions, the authors present an extension of the metaheuristic maximum likelihood approach. The performance of the parameter estimation method employed was verified through simulation tests. The fits of nonmixture, homogeneous mixture, and heterogeneous mixture distributions were evaluated through the application to a real-world case study of the extreme rainfall events of South Korea. Results indicate that the heterogeneous mixture distribution is a good alternative when sources possessing dissimilar statistical characteristics influence extreme hydrometeorological variables.
\end{abstract}

\section{Introduction}

Frequency analysis is commonly used for the prediction and risk assessment associated with hydrometeorological variables. A number of distributions have been used for the frequency analysis of extreme hydrometeorological events such as the extreme value type 1 (EV1) distribution known as "Gumbel," generalized

\footnotetext{
* Supplemental information related to this paper is available at the Journals Online website: http://dx.doi.org/10.1175/JHM-D-14-0130.s1.

Corresponding author address: Taesam Lee, Assistant Professor, Department of Civil Engineering, Gyeongsang National University, ERI, 501 Jinju-daero, Jinju, Gyeongsangnam-do 660-701, South Korea. E-mail: tae3lee@gnu.ac.kr
}

Pareto (GP) distribution, and generalized extreme value (GEV) distribution (Ahmad et al. 1988a,b; Ashkar and Ouarda 1996; Heo et al. 2008; Martins and Stedinger 2000; Russo et al. 2013; Tramblay et al. 2008). The maximum likelihood (ML), method of moments, and probability weighted moments method have been commonly used for parameter estimation of these distributions (Hamed and Rao 2010). Hassanzadeh et al. (2011) and S. Yoon et al. (2013) investigated the applicability of the metaheuristic algorithm to fit several distributions conventionally used for the frequency analysis of hydrometeorological variables. They found that the metaheuristic algorithm can efficiently estimate the parameters of the distributions employed in their study. These distributions are nonmixture distributions that have been used to carry out frequency 
analysis under the assumption that data arise from only one source. However, in reality, hydrometeorological data are often the result of multiple sources (Lee and Jeong 2014; Smith et al. 2011). Therefore, conventional nonmixture distributions can have limitations in analyzing such multisource cases, as hydrometeorological variables often do not meet their assumption.

To overcome this drawback, the use of mixture distributions was proposed for hydrometeorological variables (Carreau et al. 2009; Katz and Zheng 1999; Li and Zhang 2011; Rossi et al. 1984; Schaefli et al. 2007; Sun et al. 2009; Wójcik et al. 2006; Waylen and Woo 1983; Yue et al. 1999). Alila and Mtiraoui (2002) carried out a frequency analysis using a lognormal-lognormal mixture distribution for the Gila River in Canada, to account for the effects of the oscillations of El Niño/La Niña and channel and basin variability. Their results indicated that the application of the lognormal-lognormal mixture distribution provided a better fit to flood data than nonmixture distributions. Yoo et al. (2005) evaluated the global warming effect on daily rainfall using a mixed gamma $(\mathrm{G})$ distribution because the nonmixture distribution does not successfully represent the change in daily rainfall data. Other researchers resorted to the use of distributions for which the parameters evolve as function of time or other covariates, such as low-frequency climate oscillation indices (El-Adlouni and Ouarda 2009; ElAdlouni et al. 2007; Hundecha et al. 2008; Lee and Ouarda 2010; Masina and Lamberti 2013; Ouarda and El-Adlouni 2011). For the flood frequency analysis of the Moisie River in Canada when floods can arise from either liquid precipitation or snowmelt, Evin et al. (2011) applied the normal-normal, G-G, and EV1EV1 mixture distributions and reported that these mixture distributions provided good fit to the data. Strupczewski et al. (2012) employed an EV1-EV1 mixture distribution for the analysis of the seasonal maximum floods in Polish rivers demonstrating two mechanisms by which floods occur: heavy rainfall in the summer and melting snow in the winter. Yoon et al. (2012) and P. Yoon et al. (2013) applied EV1-EV1 and GEV-GEV mixture distributions for the frequency analysis of extreme rainfall in South Korea. Their studies showed that two extreme rainfall sources (i.e., convective rainfalls and typhoons) presented significantly different characteristics, according to the $t$ test and Wilcoxon rank-sum test.

Shin et al. (2014) investigated the performance of the metaheuristic maximum likelihood (MHML) method to fit normal-normal mixture distributions for flood frequency analysis. They concluded that the MHML successfully estimates the parameters of the normal-normal mixture distribution and provides a better performance than the expectation maximization algorithm when sample size is moderately small. These studies point out the fact that mixture distributions are good alternatives to reflect the mixture characteristics of extreme events for frequency analysis.

All these studies are limited by the assumption that the different sources of data follow an identical distribution [termed a homogeneous mixture distribution (HOM)]. It might be more rational to assume that individual sources follow different distributions [termed heterogeneous mixture distribution (HTM)] as discussed above.

HTM models have been applied in other fields. Erişoğlu et al. (2011) tested HTM models, such as exponential-G, exponential-Weibull, and G-Weibull mixture distributions, for the failure times of an oral irrigator, conventionally called dental water jet, using an expectation maximization (EM) algorithm. They used an EM algorithm to estimate the parameters of the HTM model and concluded that these HTMs successfully reproduce the characteristics of heterogeneous survival data. Carta and Ramírez (2007) proposed the truncated normalWeibull mixture distribution as a distribution for wind speed data and compared the fit of the proposed distribution with fits of Weibull and Weibull-Weibull mixture distributions for frequency analysis of wind speed data. They found that the proposed distribution gives better fit to model wind speed data than the Weibull and Weibull-Weibull distributions.

A small number of studies have attempted to apply a special case of the HTM model. Hundecha et al. (2009) and Vrac and Naveau (2007) applied the mixture distribution model composed of G and GP distributions to model rainfall amounts of nonheavy (subthreshold) and heavy events. A threshold was used to classify nonheavy and heavy rainfalls. If the rainfall amount is larger than the threshold, the rainfall event is classified as heavy rainfall. Once the threshold is defined, rainfall amounts of subthreshold and heavy rainfalls (below and above the threshold) are generated by the G and GP distributions, respectively. Since rainfall amounts generated by each distribution lead to a discontinuity of the probability at the threshold, these studies employed the HTM model in which the weight of the density component is a function of the rainfall amount. The HTM model is hence used to deal efficiently with the discontinuity in the probability of the threshold. Although the HTM employed in these studies provides an elegant way to describe rainfall amounts corresponding to subthreshold and heavy events, the HTM model employed does not represent a general formulation. Indeed, the application of the weight function violates a fundamental property 
of the general HTM model: that the probability that data belong to each distribution remains constant. In the general HTM formulation, the weight parameters represent the proportions of the whole probability of each distribution used in the mixture. Because the weight changes as a function of the rainfall amount, the proportions of the distributions are not constant in the HTM model employed.

In a pioneering study, Calenda et al. (2009) employed a normal-Gumbel mixture distribution to model the extreme floods of the River Tiber in Rome. However, this study had a relatively limited scope since it used a single station and only focused on the applicability and suitability of the HTM model. To the best of our knowledge, the general form of the HTM model has not yet been applied to the frequency analysis of extreme hydrometeorological events. Additional studies applying HTMs to a large number of stations in different hydroclimatic conditions are needed to provide more evidence concerning their usefulness and to widen the acceptance of these models and their adoption in the frequency analysis of hydrometeorological extremes. To improve our understanding of hydrometeorological variables and improve our capacity to adequately model hydrometeorological extremes, a generalized approach to HTM modeling should be developed and tested. In the current study, we develop the general form of HTM to carry out the frequency analysis for extreme hydrometeorological events. We extend the application of the MHML method to parameter estimation for the HTM case. The performance of the MHML method in fitting the HTM case is evaluated using a simulation study. It is hoped that the present work will help widen the acceptance of HTMs in the analysis of hydrometeorological extremes and accelerate their adoption in practical hydrological studies.

This paper is organized as follows. In section 2, the backgrounds of the HOM and the MHML method are described. In section 3, the description of the HTM approach is presented. In section 4 , the methodology of the simulation study and the case study application are described. In section 5 , the results of the simulation study are presented. The results of the case study are illustrated in section 6. Finally, the summary and conclusions are presented in section 7 .

\section{Background}

\section{a. Homogeneous mixture distributions}

Mixture distributions are commonly used when sample data display mixture distributional characteristics and are from multiple sources. A mixture distribution $f(y)$ is the weighted sum of the component densities $f_{i}\left(y ; \boldsymbol{\theta}_{i}\right)$ :

$$
f(y)=\sum_{i=1}^{g} \pi_{i} f_{i}\left(y ; \boldsymbol{\theta}_{i}\right)
$$

and

$$
\sum_{i=1}^{g} \pi_{i}=1
$$

where $\boldsymbol{\theta}_{i}$ is a parameter set of the $i$ th component densities and $\pi_{i}$ is the $i$ th mixing proportion or weight. The variable $\pi_{i}$ is a nonnegative quantity not larger than 1 , and $g$ is the number of mixtures.

Determining the type and number of density components of the mixture distribution is the key to successfully modeling the target variable when a mixture distribution is employed. HOM has been commonly used to model extreme events resulting from multiple sources for probability distribution models, for example, EV1-EV1, GEV-GEV, and lognormal-lognormal mixture distributions (Alila and Mtiraoui 2002; Evin et al. 2011; P. Yoon et al. 2013). Particularly, extreme value families, for example, EV1 and GEV distributions, have been frequently employed as density components of mixture distributions in modeling extreme hydrometeorological events (Evin et al. 2011; P. Yoon et al. 2013).

In the hydrological field, the record size is frequently insufficient to fit mixture distributions. The application of the GEV-GEV mixture distribution, with seven parameters, to model hydrometeorological variables would lead to inadequate results because of the lack of a sufficient number of observations. The GEV-GEV mixture distribution was hence not considered in the current study. In addition, it is difficult to estimate the shape parameter of the GEV distribution appropriately in the case of small sample sizes (El-Adlouni et al. 2007; Leclerc and Ouarda 2007; Martins and Stedinger 2000). Therefore, in the current study, the EV1-EV1 mixture distribution is employed as representative of the HOM model for the frequency analysis of extreme events. The probability density function (pdf) of the EV1 distribution is defined by

$f_{\mathrm{EV} 1}(y \mid \alpha, \beta)=\frac{1}{\beta} \exp \left[-\left(\frac{y-\alpha}{\beta}\right)-\exp \left(-\frac{y-\alpha}{\beta}\right)\right]$,

where $\alpha$ and $\beta$ are the location and scale parameters, respectively. The domain of the EV1 distribution is $-\infty<y<\infty$. The pdf of the EV1-EV1 mixture distribution is given by

$$
\begin{aligned}
f_{\mathrm{EV} 1-\mathrm{EV} 1}\left(y \mid \alpha_{1}, \beta_{1}, \alpha_{2}, \beta_{2}\right)= & \pi_{1} f_{\mathrm{EV} 1}\left(y \mid \alpha_{1}, \beta_{1}\right) \\
& +\pi_{2} f_{\mathrm{EV} 1}\left(y \mid \alpha_{2}, \beta_{2}\right) .
\end{aligned}
$$


TABLE 1. Parameters of selected densities for the simulation test. Note that the parameters of the component densities are fixed, while the weight is changed.

\begin{tabular}{|c|c|c|c|c|c|}
\hline \multirow[b]{2}{*}{$\pi_{1}$} & \multicolumn{2}{|c|}{$\mathrm{G}$} & \multirow[b]{2}{*}{$\pi_{2}$} & \multicolumn{2}{|c|}{ EV1 } \\
\hline & $a$ & $b$ & & $\alpha$ & $\beta$ \\
\hline 0.9 & & & 0.1 & & \\
\hline 0.8 & & & 0.2 & & \\
\hline 0.7 & & & 0.3 & & \\
\hline 0.6 & & & 0.4 & & \\
\hline 0.5 & 13.33 & 2.25 & 0.5 & 51.00 & 16.10 \\
\hline 0.4 & & & 0.6 & & \\
\hline 0.3 & & & 0.7 & & \\
\hline 0.2 & & & 0.8 & & \\
\hline 0.1 & & & 0.9 & & \\
\hline
\end{tabular}

\section{b. Maximum likelihood using metaheuristic application}

Maximum likelihood (ML) fits the parameters of the mixture density by maximizing the likelihood function. Equation (5) gives the likelihood function for the mixture distribution. For computational convenience, the log-likelihood function is used instead of the likelihood function. Equation (6) represents the log-likelihood function (11) of the mixture distribution:

$$
l(\boldsymbol{\pi}, \boldsymbol{\theta})=\prod_{j=1}^{n} \sum_{i=1}^{g} \pi_{i} f_{i}\left(y_{j} ; \boldsymbol{\theta}_{i}\right)
$$

and

$$
\mathbb{l l}(\boldsymbol{\pi}, \boldsymbol{\theta})=\log l(\boldsymbol{\pi}, \boldsymbol{\theta})=\sum_{j=1}^{n} \log \left[\sum_{i=1}^{g} \pi_{i} f_{i}\left(y_{j} ; \boldsymbol{\theta}_{i}\right)\right]
$$

where $n$ is the number of data points and $g$ is the number of components.

To maximize the log-likelihood function, the NewtonRaphson method has conventionally been used. Because of several drawbacks, such as local maximization, a lengthy iterative process, and the computation of complicated information matrices, the ML-Newton-Raphson combination is not conventionally used for mixture distributions (McLachlan and Peel 2000).

To address these drawbacks, an EM algorithm has been widely used for estimating the parameters of mixture distributions. However, the EM algorithm often

TABLE 2. Characteristics of the weather stations. Note that all datasets end in 2010 [e.g., No. 1 (Sokcho) 1968-2010]. Refer to Fig. 1 for the

\begin{tabular}{|c|c|c|c|c|c|c|c|}
\hline No. & Name & Altitude (m) & Length & No. & Name & Altitude (m) & Length \\
\hline 1 & Sokcho & 22.9 & 43 & 30 & Inje & 198.7 & 38 \\
\hline 2 & Chuncheon & 76.8 & 45 & 31 & Hongheon & 146.2 & 38 \\
\hline 3 & Gangneung & 26.1 & 50 & 32 & Jecheon & 263.1 & 38 \\
\hline 4 & Seoul & 85.5 & 50 & 33 & Boeun & 173.0 & 38 \\
\hline 5 & Incheon & 69.0 & 50 & 34 & Cheonan & 21.3 & 38 \\
\hline 6 & Wonju & 150.7 & 38 & 35 & Boryeong & 17.9 & 38 \\
\hline 7 & Ulleung & 220.0 & 50 & 36 & Buyeo & 11.0 & 38 \\
\hline 8 & Suwon & 34.5 & 47 & 37 & Geumsan & 170.6 & 38 \\
\hline 9 & Chungju & 113.7 & 38 & 38 & Buan & 3.6 & 38 \\
\hline 10 & Seosan & 25.2 & 43 & 39 & Imsil & 248.0 & 38 \\
\hline 11 & Uljin & 49.4 & 37 & 40 & Jeongeup & 39.5 & 38 \\
\hline 12 & Cheongju & 56.4 & 44 & 41 & Namwon & 93.5 & 38 \\
\hline 13 & Daejeon & 62.6 & 42 & 42 & Sucheon & 74.4 & 38 \\
\hline 14 & Chupungryung & 240.9 & 50 & 43 & Jangheung & 44.5 & 38 \\
\hline 15 & Pohang & 1.3 & 50 & 44 & Haenam & 4.6 & 38 \\
\hline 16 & Daegu & 57.3 & 50 & 45 & Goheung & 53.3 & 38 \\
\hline 17 & Jeonju & 61.0 & 50 & 46 & Yeongju & 210.5 & 38 \\
\hline 18 & Ulsan & 34.6 & 50 & 47 & Mungyeong & 170.8 & 38 \\
\hline 19 & Gwangju & 74.5 & 50 & 48 & Yeongdeok & 41.2 & 38 \\
\hline 20 & Busan & 69.2 & 50 & 49 & Uiseong & 82.6 & 38 \\
\hline 21 & Tongyeong & 30.8 & 43 & 50 & Gumi & 47.4 & 38 \\
\hline 22 & Mokpo & 37.4 & 50 & 51 & Yeongcheon & 93.3 & 38 \\
\hline 23 & Yeosu & 73.3 & 50 & 52 & Geochang & 221.4 & 38 \\
\hline 24 & Wando & 27.7 & 37 & 53 & Hapcheon & 33.0 & 38 \\
\hline 25 & Jeju & 19.9 & 50 & 54 & Miryang & 10.7 & 38 \\
\hline 26 & Seogwipo & 50.4 & 50 & 55 & Sancheong & 138.7 & 38 \\
\hline 27 & Ganghwa & 46.2 & 38 & 56 & Geoje & 44.5 & 38 \\
\hline 28 & Yangpyeong & 47.4 & 38 & 57 & Namhae & 43.2 & 38 \\
\hline 29 & Icheon & 90.0 & 38 & & & & \\
\hline
\end{tabular}
geographic locations of stations. 


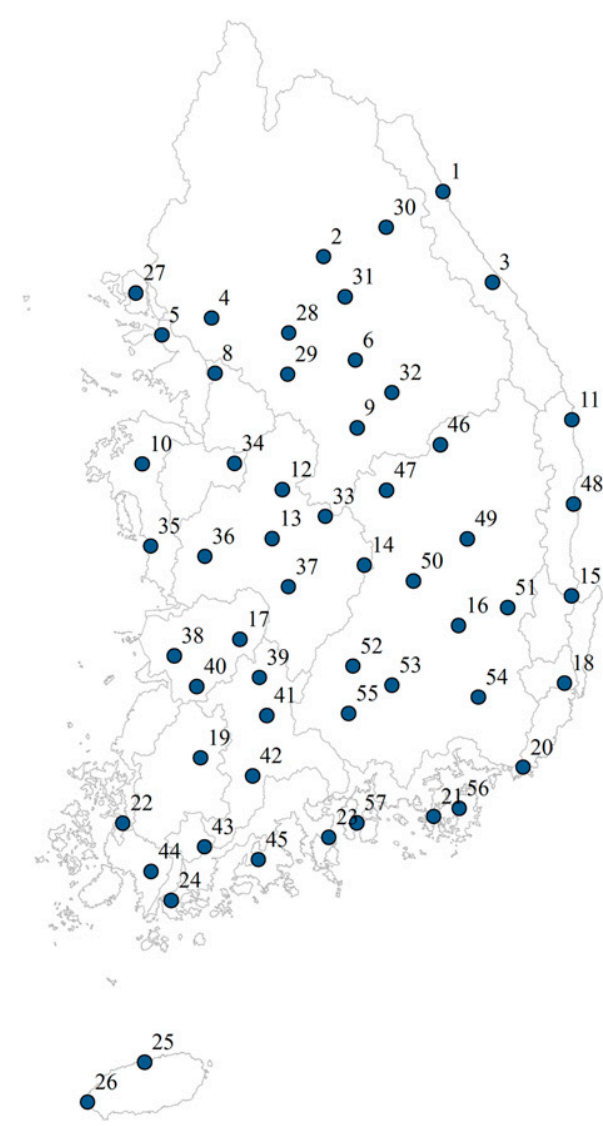

FIG. 1. Geographic locations of weather stations. The names of the stations corresponding to the numbers are listed in Table 2.

estimates inaccurate weight parameters when the weight parameter value is small. In addition, it frequently diverges when the number of observations is insufficient. In the hydrometeorological field, the record size is often so small that the data are insufficient to obtain stable estimates of the parameters of mixture distributions using the EM algorithm.

Shin et al. (2014) adapted the MHML algorithm to overcome these drawbacks and estimate the parameters of mixture models. Metaheuristic algorithms are optimization methods that iteratively try to improve a candidate solution with regard to a given measure of quality. This approach yields better performances than the EM algorithm when estimating the parameters of mixture distributions, especially in the estimation of weight parameter. In particular, this approach shows a significant advantage when the number of data points is very low (even below 50). Therefore, in the current study, we tested and applied a metaheuristic algorithm to estimate the parameters of the HTM.

Various metaheuristic applications have been suggested, such as genetic algorithm (GA), particle swarm optimizations and harmony searches (Geem et al. 2001;
Goldberg 1989; Kennedy and Eberhart 1995). In the current study, a GA was used. As a representative of metaheuristic algorithms, GA successfully finds the optimal solution by emulating realistic processes of evolution.

A GA consists of genes, individuals, populations, and generations. Individuals have genes that represent their own original characteristics. A generation consists of a predefined number of individuals, which are called the population. Individuals in the initial generation, that is, parents, produce offspring through selection, crossover, and mutation. An objective function selects the offspring that will survive and become the parents of the next generation. New parents produce new offspring through genetic combination; some of the new offspring are mutated. These offspring become the next generation. This procedure is repeated until parents and offspring possess nearly identical fitness.

GA adapts a population while many other optimization methods work with a single point. Hence, in GA, the probability of finding local or wrong optimal solutions is reduced. In addition, the computation of information matrices is not necessary because maximization by a GA is only necessary to derive the objective function, as it would be for the ML method. However, in the GA, standard errors of parameter estimates based on the asymptotic approach cannot be applied because GA does not rely on the information matrix. In the current study, the log-likelihood function, Eq. (6), is employed as an objective function in GA.

\section{Heterogeneous mixture distributions}

Many studies employed mixture distributions to model extreme events resulting from multiple sources that show heterogeneous behaviors. These studies assumed that the different sources present the same type of distribution. However, it is rational to assume that the multiple sources showing heterogeneous behaviors follow different distributions.

The $\mathrm{G}$ distribution has been commonly used to generate rainfall amounts in stochastic simulations (Chen et al. 2010; Kleiber et al. 2012; Wilks and Wilby 1999). The amount of rainfall in subthreshold events and heavy rainfalls presents different behaviors. The $\mathrm{G}$ distribution cannot successfully reproduce the amounts of heavy rainfall events (Cameron et al. 2000; Katz et al. 2002). To overcome this limitation of the $G$ distribution, Hundecha et al. (2009) and Vrac and Naveau (2007) employed the G-GP mixture distribution to model the amount of rainfall in stochastic simulation. In these studies, it was assumed that the distributions of rainfall for dry (nonheavy rainfall) and wet (heavy rainfall) spells are different. Both studies used the G and GP 

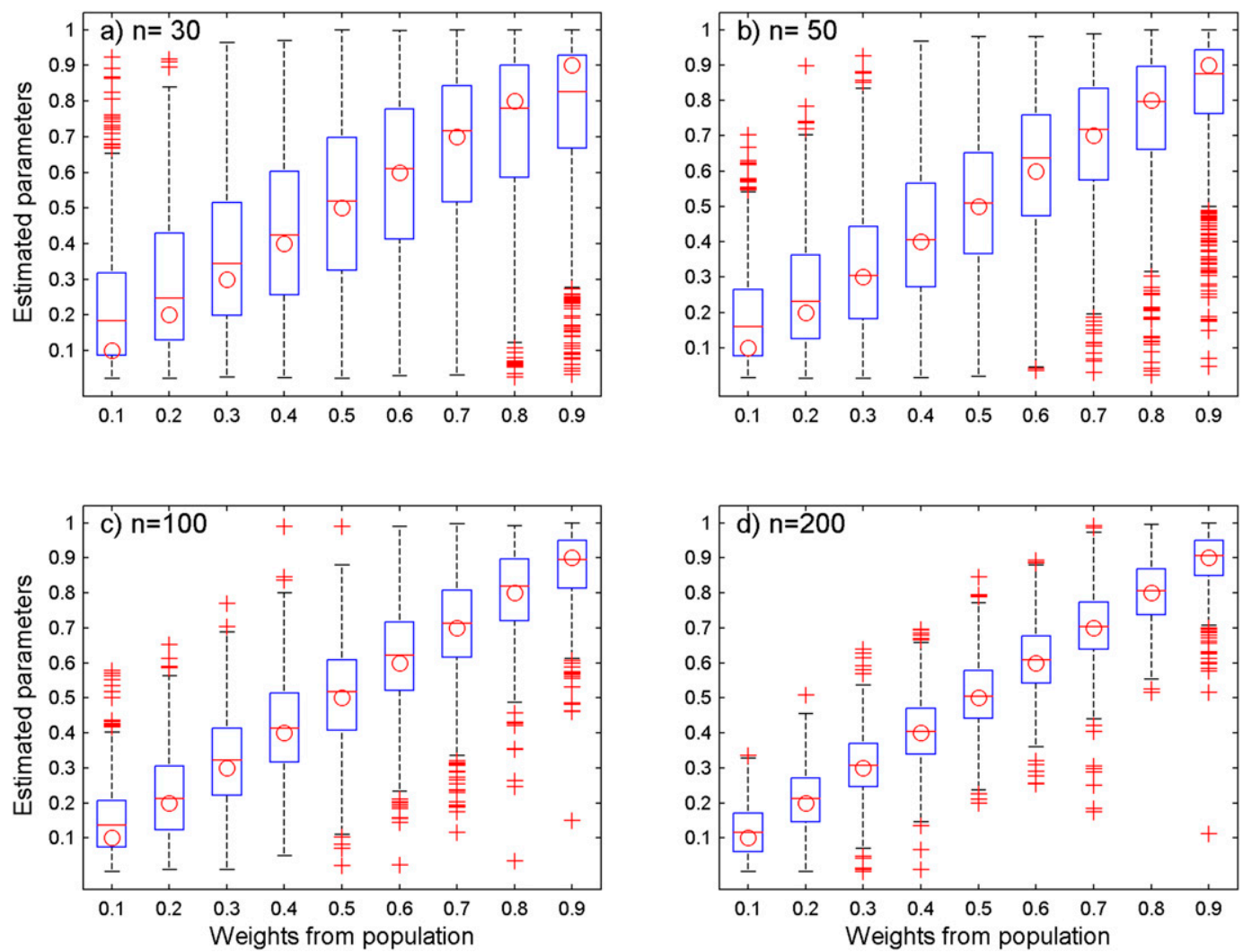

FIG. 2. Box plots of the estimated weight $\pi_{2}$ of the EV1 distribution for different record lengths: (a)-(d) $n=30$, 50,100 , and 200, respectively. The red circles represent the weight of the population. The horizontal lines inside the boxes show the median of the data. The data beyond the whiskers $(1.5 \times \mathrm{IQR})$ are indicated by a plus marker $(+)$. The plus markers and the red horizontal lines indicate outliers and the median of estimates, respectively. This same convention is used in the other box plots.

distributions to model the amounts of dry events and heavy rainfalls, respectively.

In the current study, the G and EV1 distributions are also employed for density components of the HTM. As previously mentioned, the $\mathrm{G}$ distribution has commonly been employed to model rainfall events (Ashkar and Ouarda 1998; Chen et al. 2010; Katz 1999; Phien and Jivajirajah 1984; Yoo et al. 2005; Yue et al. 2001). The EV1 distribution has often been used for the frequency analysis of extreme events in the hydrometeorological field (Escalante-Sandoval 1998; Evin et al. 2011; Ouarda and Shu 2009; Park et al. 2011; Vogel 1986; Yoon et al. 2012). In addition, because the EV1 distribution is a limiting case of the $\mathrm{G}$ distribution, the mixture of these two distributions might successfully reproduce a broad range of behaviors for hydrometeorological variables. The pdf of the G-EV1 mixture distribution is given by

$$
\begin{aligned}
f_{\mathrm{G}-\mathrm{EV} 1}(y \mid a, b, \alpha, \beta)= & \pi_{1} f_{G}(y \mid a, b) \\
& +\pi_{2} f_{\mathrm{EV} 1}(y \mid \alpha, \beta) .
\end{aligned}
$$

The pdf of the $\mathrm{G}$ distribution is given by

$$
f_{G}(y \mid a, b)=\frac{1}{a^{b} \Gamma(b)} y^{b-1} e^{-(y / a)},
$$

where $a$ and $b$ are the scale and shape parameters, respectively. The domain of the $\mathrm{G}$ distribution is $y>0$.

\section{Study methodology}

\section{a. Simulation tests}

The MHML algorithm is employed to estimate the parameters of the HTM, that is, G-EV1 mixture distributions. The performance of the proposed MHML algorithm is tested for the HTM model through the designed simulation study as follows.

First, the parameters of the population distribution are estimated. The weights $\pi_{2}$ of the EV1 distribution take values from 0.1 to 0.9 with increments of 0.1 when the parameters of the density components are fixed. In the present study, it is assumed that the EV1 and G distributions dictate the behaviors of the right and left tails, respectively, of the HTM. Therefore, the mean of the EV1 distribution should be larger than the mean of 

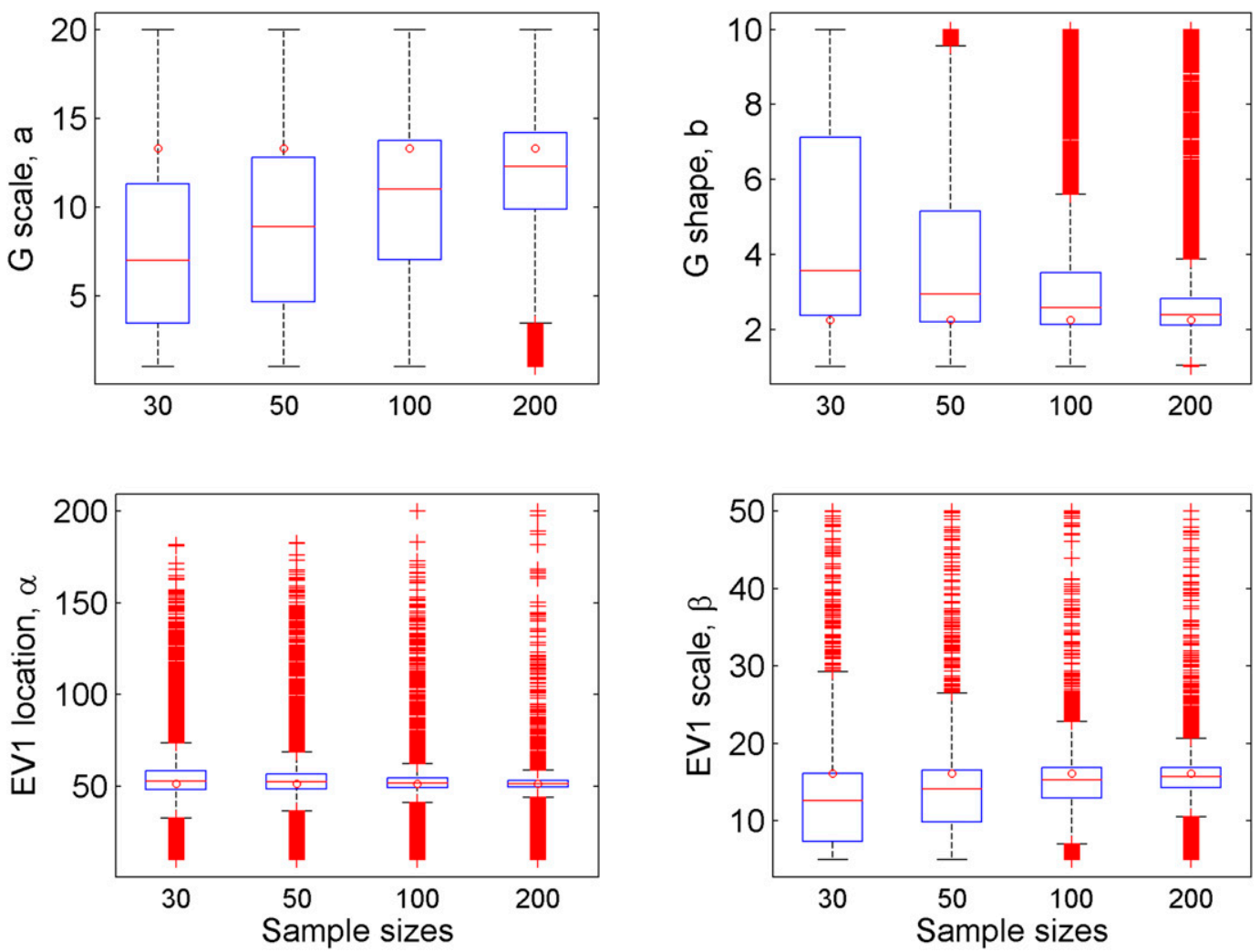

FIG. 3. As in Fig. 2, but for estimates of simulated (box plots) and target (circles) parameters from the tested mixture distributions [see Eq.(7)].

the $\mathrm{G}$ distribution. In the current study, the means of EV1 and G distributions are 60 and 30, respectively, and these distributions have the same standard deviation of 20. The parameters of the mixture distribution used for simulation tests are presented in Table 1.

Four sample sizes $(30,50,100$, and 200) are used to test the performance of the MHML algorithm. One thousand sample sets are generated for each sample size. The parameters are estimated from generated sample sets using the MHML algorithm. To verify the performance of the model, the estimated parameters are compared to the parameters used to generate the sample sets.

\section{b. Case study}

The sources of extreme rainfall in South Korea are convective storms (often called Changma) and typhoons. Heavy rainfall over South Korea usually results from individual convective storms or mesoscale convective storm systems. On average, three typhoons also affect the total rainfall of South Korea every year. These typhoons are often accompanied by severe heavy rainfall and extremely high wind speeds (Kwon et al. 2008). Because of the influence of the two types of systems on heavy rainfalls, a mixture distribution might be appropriate to model the rainfall extremes in South Korea. Furthermore, rainfall events resulting from the two systems have different statistical characteristics, making them good candidates for the application of mixture distributions. Annual maximum rainfalls of South Korea are employed for the case study.

The annual maximum rainfalls $(24 \mathrm{~h})$ of 57 weather stations managed by the Korea Meteorological Administration (KMA) are used. Table 2 presents the list of the weather stations, and Fig. 1 illustrates their location. Figure 1 indicates that the stations employed are well distributed over the whole area of South Korea. Rainfall record lengths range between 38 and 50 years. The number of observations in some stations is relatively small. However, for most stations, record lengths are considered sufficiently large to fit the employed mixture distributions using the MHML algorithm.

Three distributions, EV1, EV1-EV1, and G-EV1, are applied based on the previous discussion. Particularly, because the EV1 distribution has strongly been recommended for the frequency analysis of extreme rainfalls in South Korea, we select it as the nonmixture 

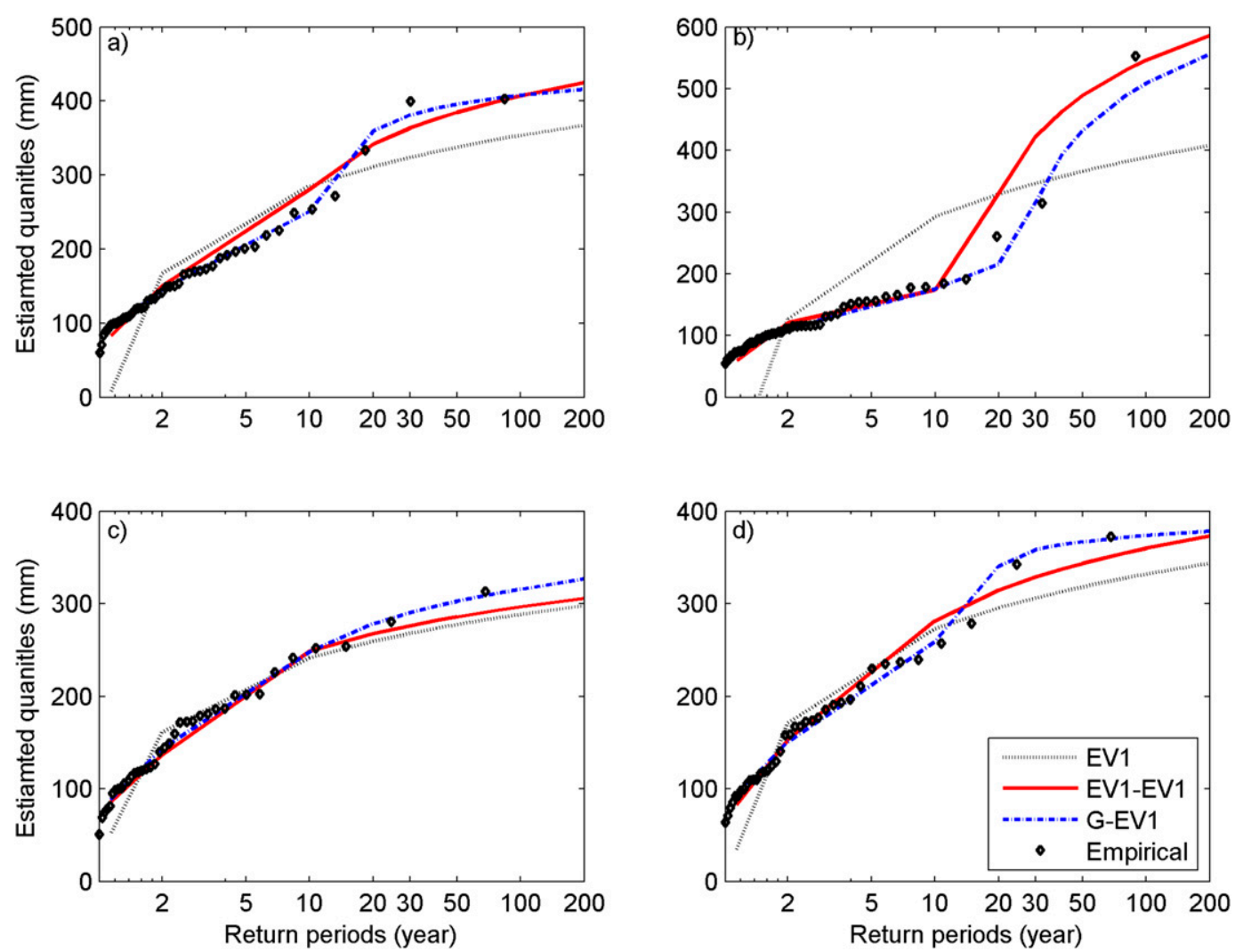

FIG. 4. Plots of estimated quantiles for (a) Suwon (station 8), (b) Pohang (station 15), (c) Inje (station 30), and (d) Hongcheon (station 31) using EV1 (dotted line), EV1-EV1 mixture (solid line), and G-EV1 mixture (dashdotted line) distributions.

distribution (Kim et al. 2000; Nadarajah and Choi 2007). The G-EV1 mixture distribution is chosen to represent the HTM. For comparison purposes, the EV1-EV1 mixture distribution is used as a representative of HOM. This distribution has been proven to represent a reasonable approximation for extreme rainfall events in South Korea (Yoon et al. 2012). The goodness of fit (GOF) of the selected distributions is compared through the evaluation criteria listed below.

\section{c. Evaluation criteria}

To measure the fitness of the various candidate distributions, three criteria-the log-likelihood, the root-meansquare error (RMSE), and the Akaike information criterion (AIC) - are used. The log-likelihood and the RMSE measure the fit of the selected distributions, while the AIC measures the performance of a given model while pondering parsimony considerations (Akaike 1974).

The log-likelihood is computed through Eq. (6). Equations (9) and (10) are the specific equations for the RMSE and AIC, respectively:

$$
\mathrm{RMSE}=\sqrt{\frac{\sum_{i=1}^{n}\left(\hat{Q}_{i}-Q_{i}\right)^{2}}{n}}
$$

and

$$
\mathrm{AIC}=2 k-2 \ln \left[\prod_{j=1}^{n} \sum_{i=1}^{g} \pi_{i} f_{i}\left(y_{j} ; \boldsymbol{\theta}_{i}\right)\right],
$$

where $Q_{i}$ is an empirical quantile, $\hat{Q}_{i}$ is a computed quantile, $n$ is the number of data points, and $k$ is the number of distribution parameters. To compute the empirical quantiles, the Gringorten plotting formula is used (Arnell et al. 1986).

The AIC is a relatively severe measure for mixture distributions because the AIC penalizes the number of estimated parameters in the model [see $k$ in Eq. (10)]. The AICs of each mixture distribution include six penalties as these distributions are penalized for their additional parameters in comparison to the EV1 distribution [Eq. (10)].

Box plots are also used for an overview of the performance of the generated data. The boxes display the 

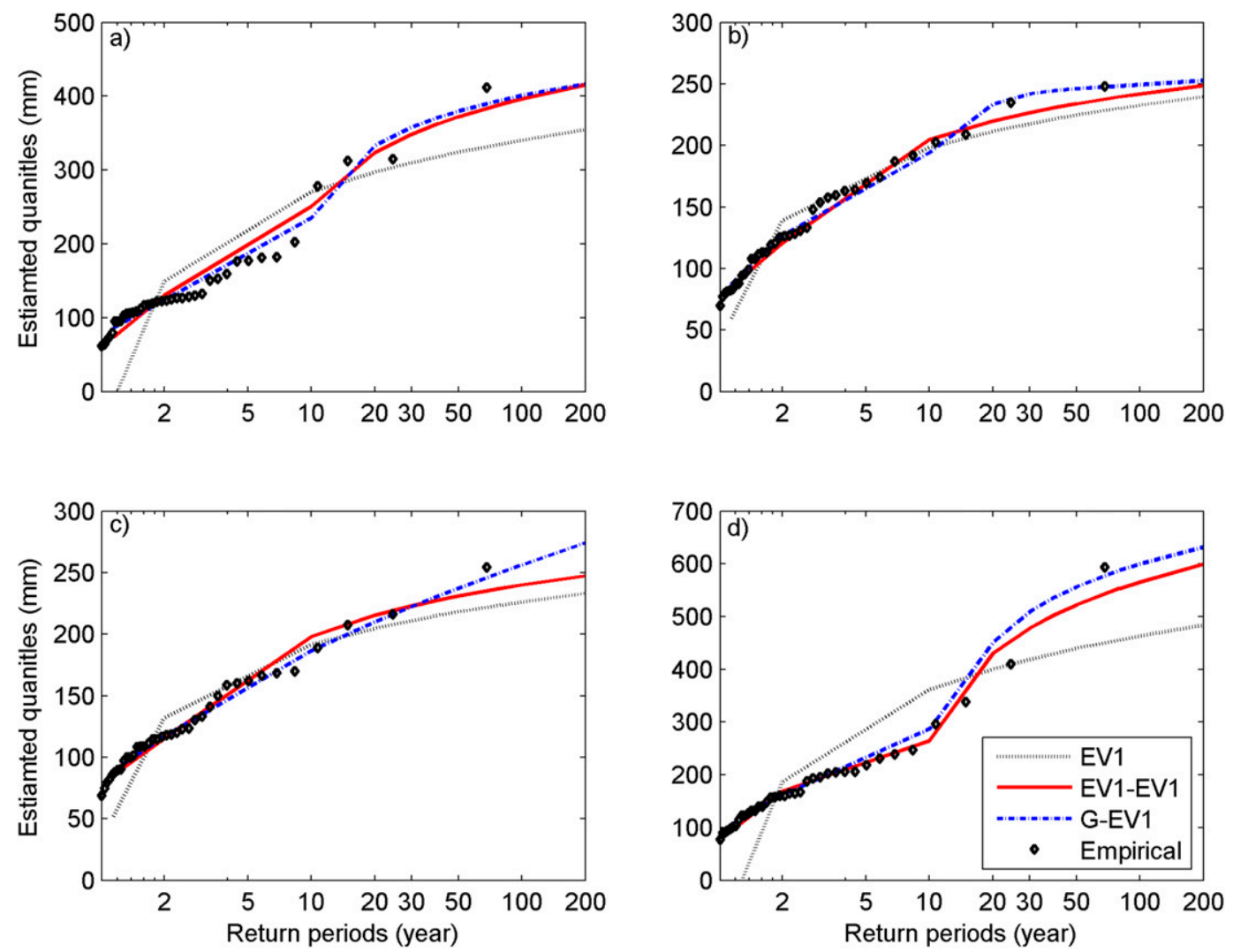

FIG. 5. As in Fig. 4, but for (a) Boeun (station 33), (b) Imsil (station 39), (c) Jeongeup (station 40), and (d) Goheung (station 45).

interquartile range (IQR) and the whiskers extending to $1.5 \times$ IQR. The horizontal lines inside the boxes show the median of the data. The data beyond the whiskers $(1.5 \times \mathrm{IQR})$ are indicated by a plus marker $(+)$. Observation statistics are presented with circles.

\section{Simulation results}

Figure 2 presents the box plots of the estimated weight parameters $\pi_{2}$ of the EV1 density components for all sample sizes. Note that the weight parameter of the $G$ density is simply equal to $\pi_{1}=1-\pi_{2}$ and is not shown here.

Figure 3 presents the estimates of the simulated and target parameters from the tested mixture distributions for different sample sizes. Figure 3 indicates that the population weight parameters are located inside the box for all test cases $\left(\pi_{2}=0.1-0.9\right)$. This implies that the MHML algorithm successfully estimates the weight of the HTM (i.e., G-EV1 mixture distribution) for various sample sizes. Forty data entries can be an insufficient number to reflect the mixture distribution, as noted by Evin et al. (2011); simulation results indicate that the MHML algorithm accurately estimates the weight parameters when the record length is short (i.e., 30 and 50).
Figure 3 indicates also that the estimated parameters of the EV1 and G distributions are narrowly located near their target values. Results indicate that the MHML algorithm can provide a good estimate of the location parameters of the EV1 density component. In addition, the population scale parameter is located inside the boxes of estimated scale parameters for EV1, and the population shape parameter is located inside the boxes of shape parameter estimates for $G$ distributions except for the case of a sample size of 30 . We can hence conclude that the MHML algorithm successfully estimates the EV1 population parameters. However, the scale parameter $a$ of the G density component is rather underestimated when the record length is too short (i.e., $n$ is lower than 50). The results of simulation-based comparison of the EM and MHML methods are included in the supplemental material.

\section{Case study results \\ a. Quantile comparisons}

Eight stations are chosen to illustrate the overall performance of the selected distribution models. The locations of these stations would cover the overall area of South 

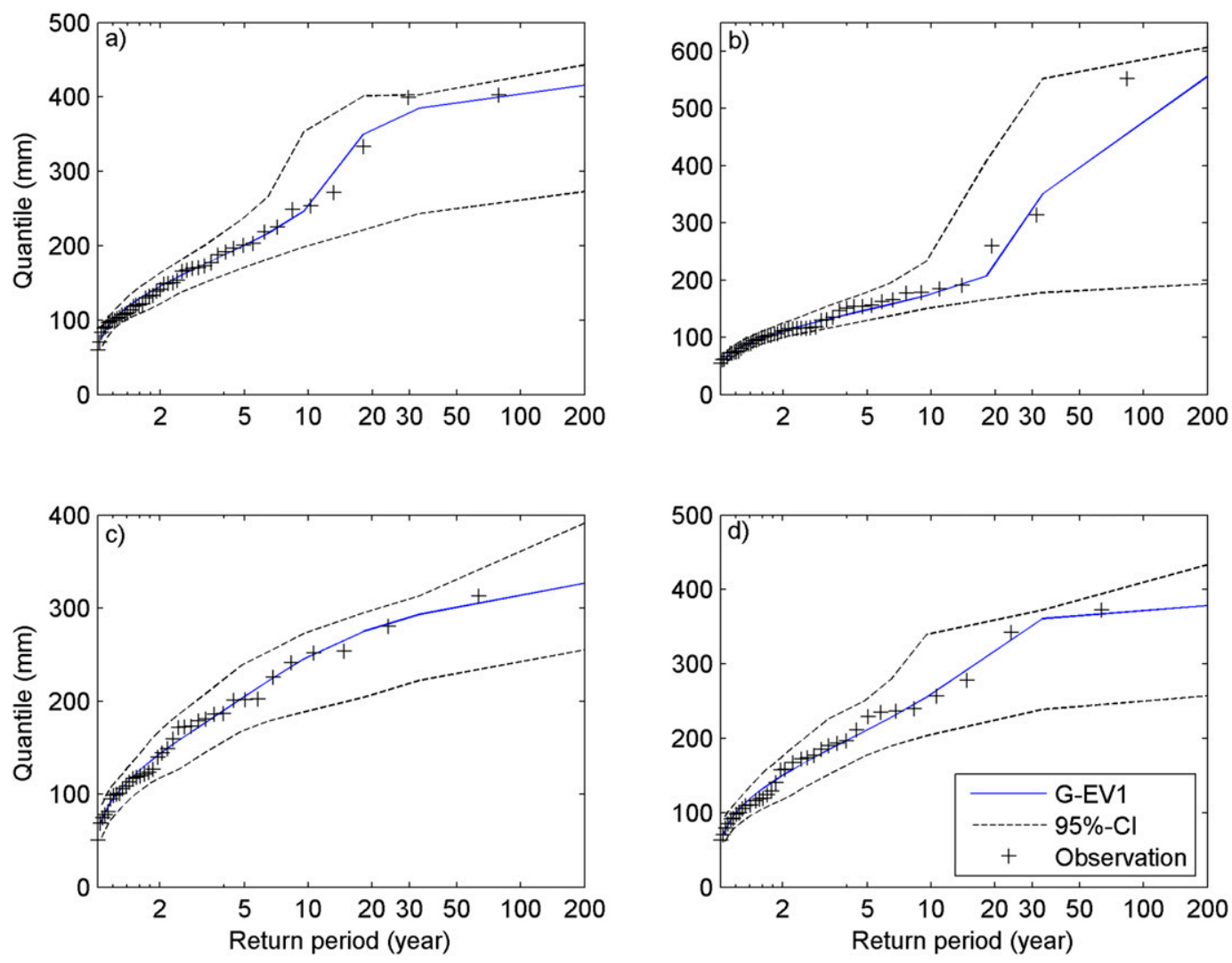

FIG. 6. Plots of observation, quantiles of G-EV1 mixture distribution, and CIs corresponding to the $95 \%$ significance level for (a) Suwon, (b) Pohang, (c) Inje, and (d) Hongcheon.

Korea. Their quantiles are presented in Figs. 4 and 5. In most cases, the quantiles estimated by the mixture distributions closely followed the empirical quantiles. On the other hand, the EV1 distribution does not successfully estimate the quantile values. In particular, the mixture distributions show remarkable improvements in quantile estimation for high- and low-return periods (the upper and lower tails of the distribution) over the nonmixture distribution (EV1). Only Boeun shows clear characteristics of mixture distributions. However, the authors consider that three stations, such as Suwon, Pohang, and Hongcheon, also show characteristics of mixture distribution, although this is not as clear as in the case of Boeun.

For example, in the empirical quantiles of Fig. $4 b$, the sections corresponding to the low-return periods and high-return periods show different slopes and behaviors
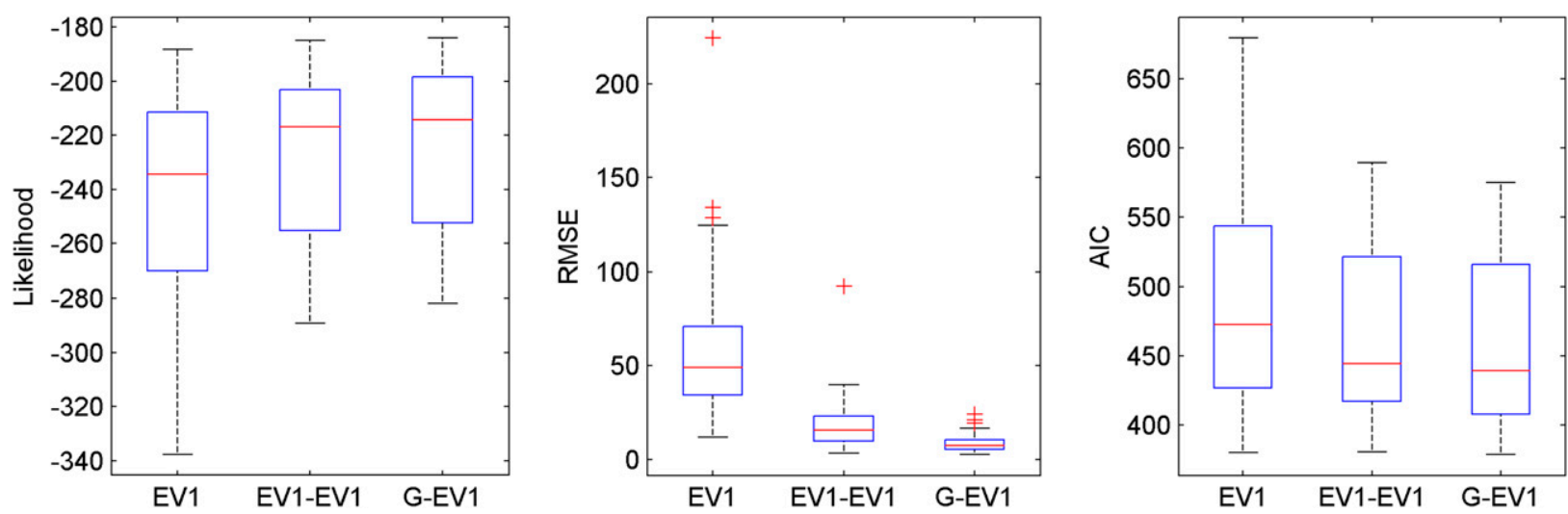

FIG. 7. Box plots of the three performance measures (left) likelihood, (center) RMSE, and (right) AIC of EV1 (nonmixture), EV1-EV1 (HOM), and G-EV1 (HTM) distributions for all 57 stations in South Korea. 
a)
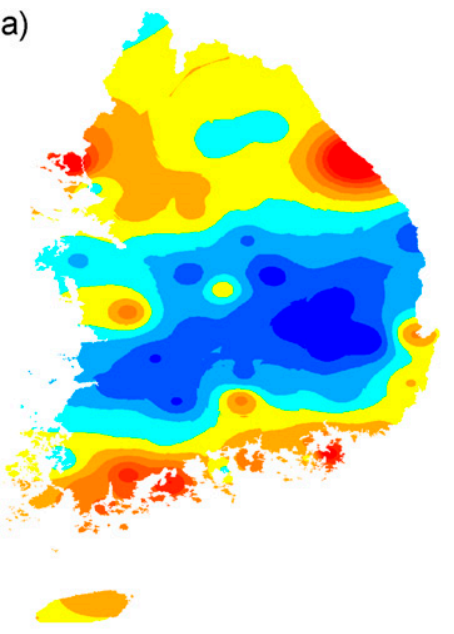

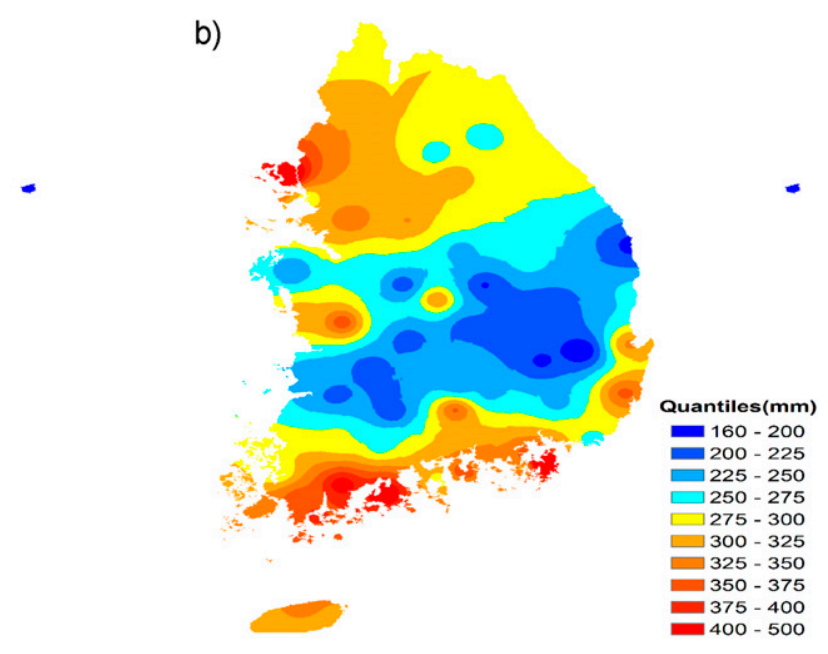

c)

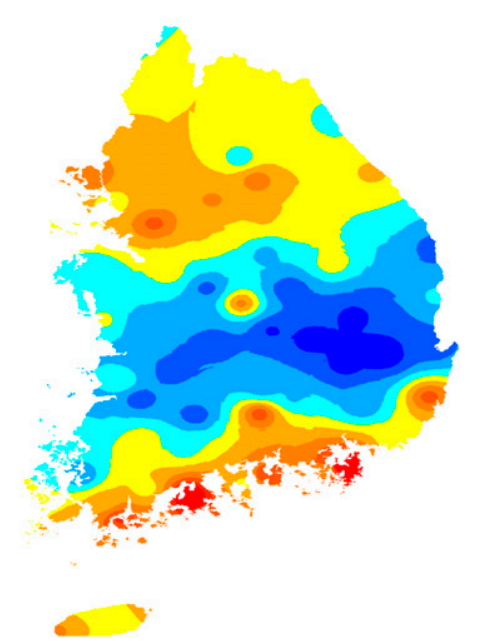

FIG. 8. Spatial distribution of the estimated quantiles for (a) EV1, (b) EV1-EV1 mixture, and (c) G-EV1 mixture distribution in South Korea for the 20-yr return period. The color quantiles range from dark blue for 160-200 $\mathrm{mm}$ to dark red for $400-500 \mathrm{~mm}$ in varying intervals.

(dog-leg shape). In this case, the nonmixture distribution (i.e., EV1) does not successfully reflect the mixture distributional characteristics of the annual maximum rainfalls. Figures 4 and 5 show that the EV1 underestimates quantiles corresponding to high-return periods. This is critical for the design of hydraulic structures, where an underestimation could result in an under design of the structures, rendering them more vulnerable to the actual extreme flooding events that will occur (Ahmad et al. 1988a,b; Chebana and Ouarda 2008; Rossi et al. 1984; Singh et al. 2005).

In contrast to nonmixture distributions, the mixture distributions successfully reproduce the statistical characteristics of the data. Figures 4 and 5 show that the estimated quantiles corresponding to high-return periods from mixture distributions closely follow the empirical quantiles. This is particularly true for the four heaviest rainfalls resulting from typhoons at Goheung station presented in Fig. 5d. These typhoons caused historic floods in South Korea that could be considered as outliers. Modeling these very heavy rainfall events is difficult because their statistical characteristics are different from the other events. It is observed that the EV1 distribution of the HTM model successfully characterizes the heavy rainfalls resulting from typhoons. These results support the hypothesis that annual maximum rainfalls in a number of South Korean stations show mixture characteristics. In general, the HTM model better reflects the behavior of quantiles corresponding to high-return periods than the HOM model.

In the present study, nonparametric bootstrapping (Hu et al. 2015; Overeem et al. 2008) is applied to obtain the standard error of the HTM. McLachlan and Peel 

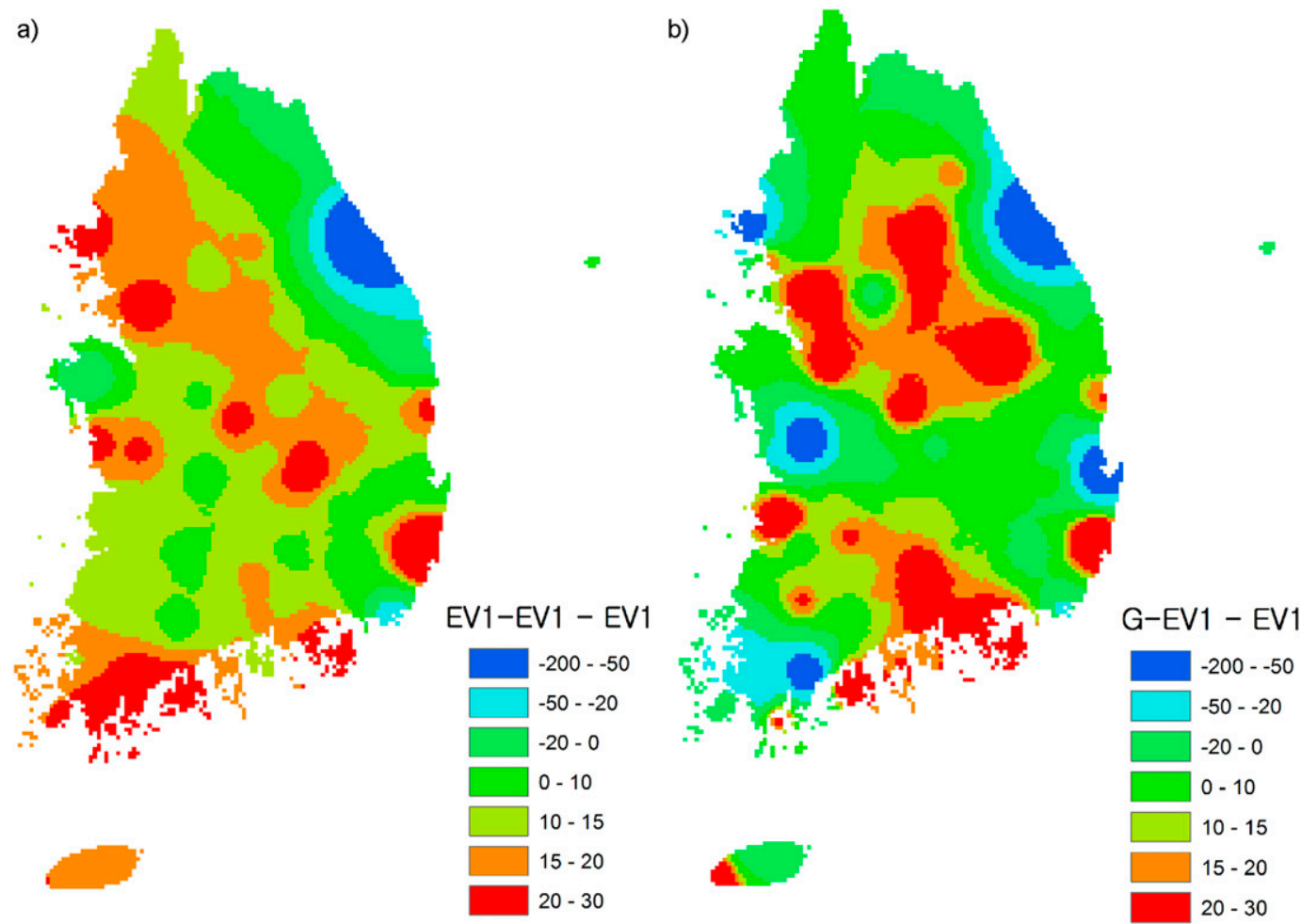

FIG. 9. Differences between the quantiles of nonmixture distributions (EV1) and the quantiles of mixture distributions (a) EV1-EV1 and (b) G-EV1 for the 20-yr return period. The differences were computed by subtracting the quantiles of the nonmixture distributions from the quantiles of the mixture distributions.

(2000) reported that approximately 800 resampled datasets are required to obtain a reliable standard error from the bootstrap method. Therefore, in the present study 1000 series were bootstrapped (i.e., randomly sampled with replacement) from the original sample dataset. Parameters of the HTM for the bootstrapped dataset are estimated by the MHML. Confidence intervals (CIs) of 2.5 and 97.5 percentiles are obtained by the estimated parameters of the bootstrapped series. Estimated CIs corresponding to the $95 \%$ significance level for four stations are presented in Fig. 6. These results indicate that the parameters of the G-EV1 mixture distribution are successfully estimated by the MHML method. The lower bound is located at a very low value for Suwon, Pohang, and Hongcheon stations. The lower bounds of these three stations come from the gamma distribution as the value of the weight parameter of the gamma distribution is nearly one.

\section{b. Goodness of fit}

Quantiles estimated by mixture distributions tend to be higher than quantiles corresponding to nonmixture distributions. However, this does not guarantee better fit. Goodness of fit of the employed distributions is measured using the likelihood, the RMSE, and the AIC criteria.
The G-EV1 mixture distribution shows the largest likelihood and the smallest RMSE in all stations but Sucheon (station 42), where the EV1-EV1 mixture distribution shows the largest likelihood and the smallest RMSE. Although the two mixture distributions have the same number of parameters, the G-EV1 mixture distribution (HTM) leads to a better fit than the EV1EV1 mixture distribution (HOM) for the annual maximum rainfalls in South Korea.

The first two GOF measures (i.e., RMSE and likelihood) do not take into consideration the number of parameters of the distribution. Therefore, the AIC, which has a penalty term for the number of parameters, is used instead. Despite the AIC's higher penalty for the G-EV1 mixture distribution than the EV1 distribution, the G-EV1 mixture distribution demonstrates the best fit in all stations except for Sucheon and Youngcheon (station 51). These results indicate that the HTM is a more appropriate model for the frequency analysis of extreme rainfalls in South Korea. This conclusion takes into consideration the fact that the two sources of extreme rainfall in South Korea (i.e., Changma and typhoons) may follow different distributions.

Figure 7 illustrates box plots of the three performance measures of employed distributions for all 54 stations in 

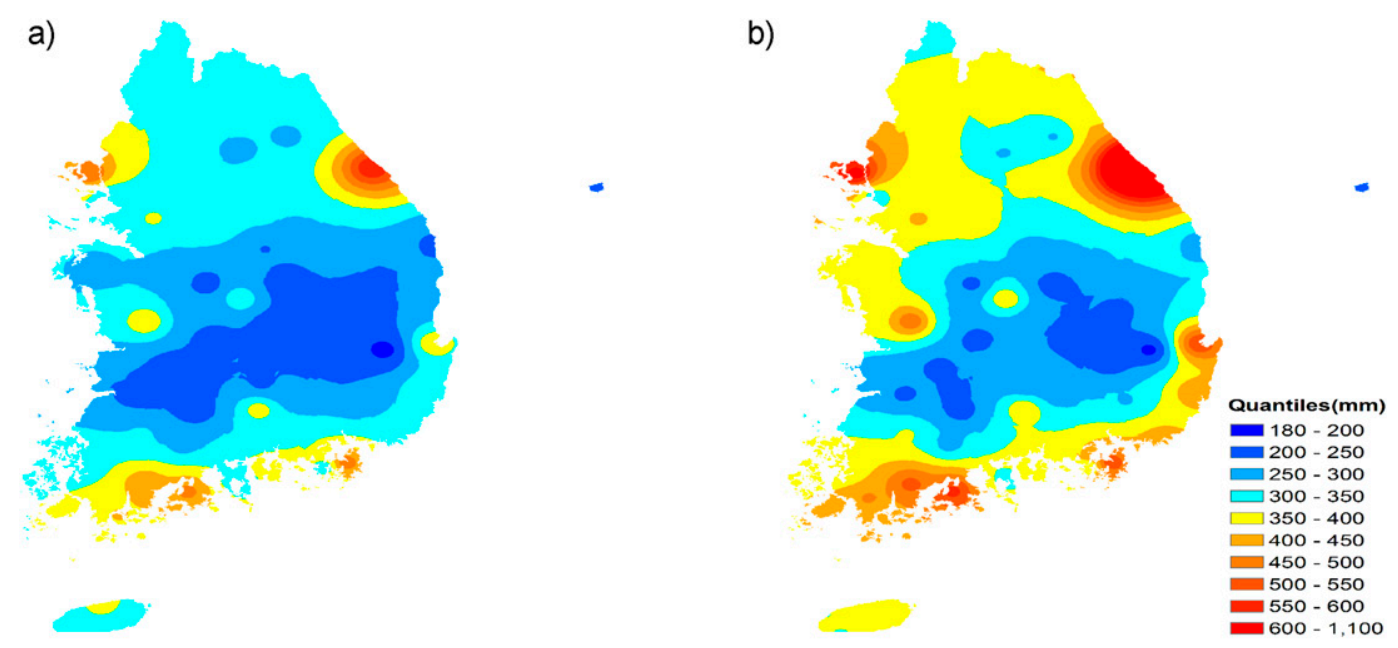

c)

FIG. 10. As in Fig. 8, but for the 100-yr return period. The color quantiles range from dark blue for 180-200 $\mathrm{mm}$ to dark red for $600-1100 \mathrm{~mm}$ in varying intervals.

South Korea. Figure 7 indicates that the EV1 distribution leads to a poor fit according to all performance measures. Particularly, the median of the RMSEs for the EV1 distribution is much larger than the median of the RMSEs for all mixture distributions, with the exception of one outlier for the EV1-EV1 mixture distribution. The G-EV1 mixture distribution leads to better results than the EV1-EV1 mixture distribution according to all performance measures.

\section{c. Spatial distribution of quantiles}

Figure 8 presents the spatial distribution of the estimated quantiles when the return period is 20 years. To investigate the differences between nonmixture and mixture distributions, the quantiles of nonmixture distributions (EV1) are subtracted from the quantiles of mixture distributions. Figure 9 presents the differences between the quantiles of the nonmixture and mixture distributions when the return period is 20 years. In the inland areas, the quantiles of the G-EV1 mixture distribution are higher than those of the EV1 distribution while the quantiles of the G-EV1 mixture distribution are lower in coastal areas.

As mentioned above, estimation of quantiles corresponding to high-return periods such as 100 and 200 years is critical for the design of hydraulic structures. For instance, the 100- and 200-yr return periods are commonly used as design criteria of hydraulic structures in South Korea. The spatial variability of these quantiles is further investigated. Figure 10 presents the spatial distribution of the estimated quantiles for the 100-yr return period. Figure 10 indicates that the quantiles of the EV1-EV1 mixture distribution are the largest in most areas. It is observed that the quantiles of the mixture distributions in coastal areas are much higher than the quantiles of the nonmixture distributions in the same area. 
a)

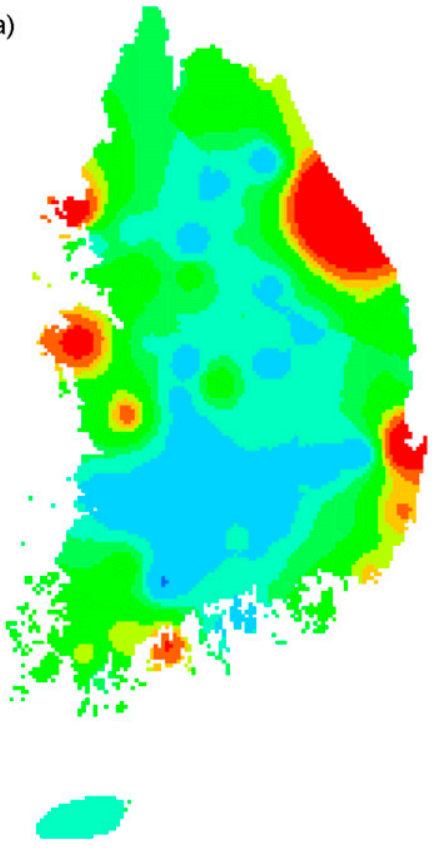

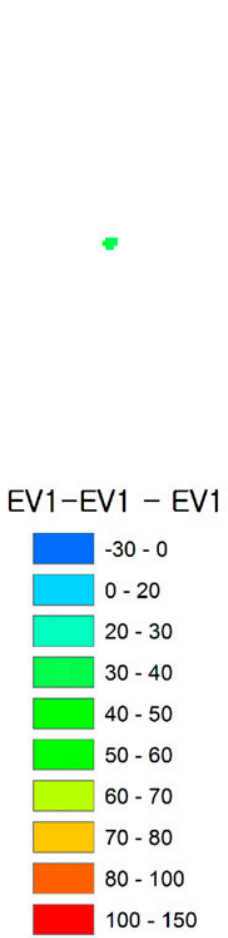

b)

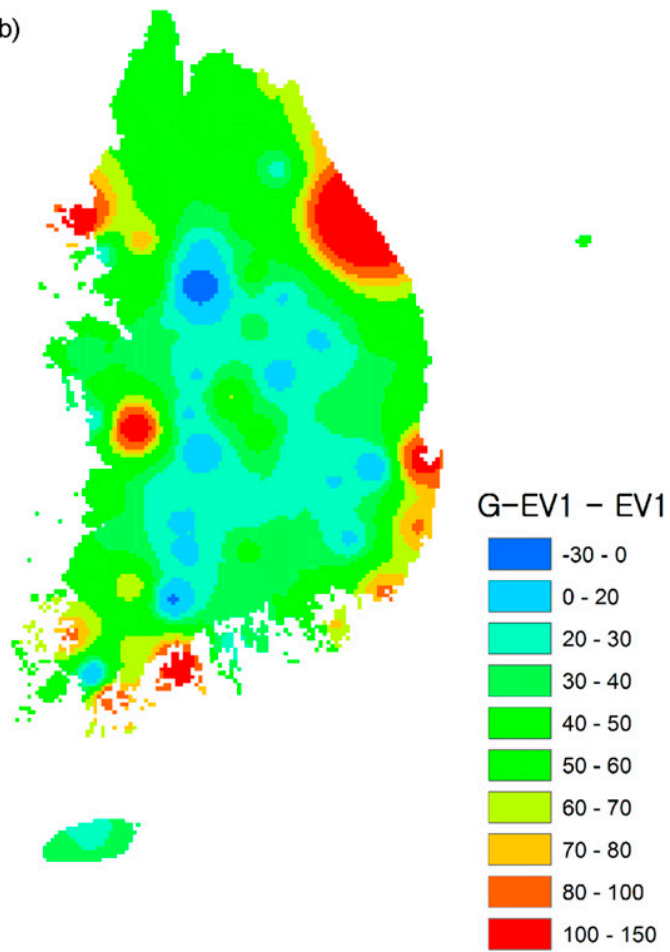

FIG. 11. As in Fig. 9, but for the 100-yr return period.

To compare the quantiles of the nonmixture and mixture distributions, the quantiles of the nonmixture distributions are subtracted from the quantiles of mixture distributions for the 100-yr return period. Figure 11 illustrates the difference between the quantiles of nonmixture and mixture distributions. The results show that quantiles of mixture distributions are larger than those of nonmixture distributions in most areas. In particular, the quantiles of the mixture distributions in coastal areas increase remarkably. We suspect that stations in coastal areas are generally more directly impacted by typhoons; in such cases, mixture characteristics are strongly indicated. In addition, the majority of the largest extreme rainfalls are caused by typhoons. Because nonmixture distributions could not consider the two different sources of rainfall, their quantiles do not successfully reflect this effect.

Figure 12 presents the spatial distribution of the estimated quantiles when the return period is 200 years. Figure 12 indicates that the quantiles of the G-EV1 mixture distribution are the largest in most areas. Figure 13 presents the differences between the quantiles of nonmixture and mixture distributions for comparison purposes. The results indicate that the quantiles of the mixture distributions are larger than those of the nonmixture distribution in most areas. While the differences between the quantiles of the nonmixture and the HOM (i.e., EV1-EV1 mixture) distributions are similar to those corresponding to the $100-y r$ return period, the differences between the quantiles of the nonmixture distribution and the HTM (i.e., G-EV1) distribution are slightly higher than the differences at the 100 -yr return period.

\section{Summary and conclusions}

The multitude of sources of hydrometeorological extreme events represents a good argument for the use of mixture distributions. Previously, it has commonly been assumed that the various sources of data have identical types of density functions when the mixture distribution is applied. In the present work, we extend the mixture distribution framework to the case when the various sources of hydrometeorological variables possess different types of densities.

Therefore, here, we develop the HTM to model extreme events of hydrometeorological variables. We test the EV1-EV1 and G-EV1 mixture distributions, representing the HOM and HTM models, respectively. The EV1 is used as a representative of nonmixture distributions. The MHML algorithm is extended to estimate the parameters of mixture distributions. The MHML algorithm is tested to estimate the parameters of the HTM (i.e., G-EV1 mixture distribution), and results 
a)

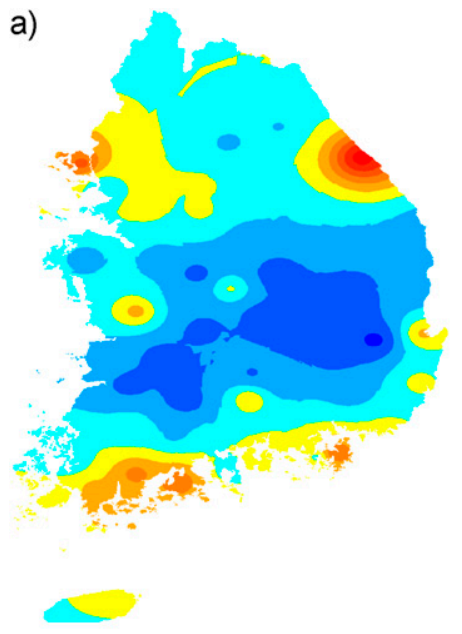

b)

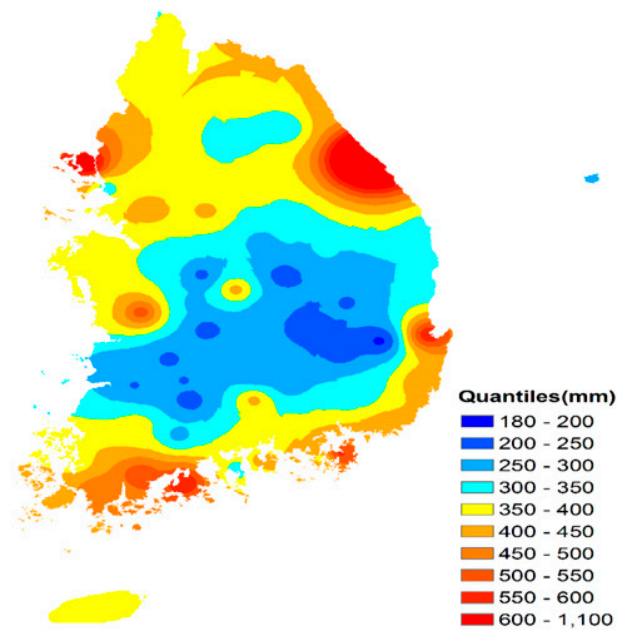

c)

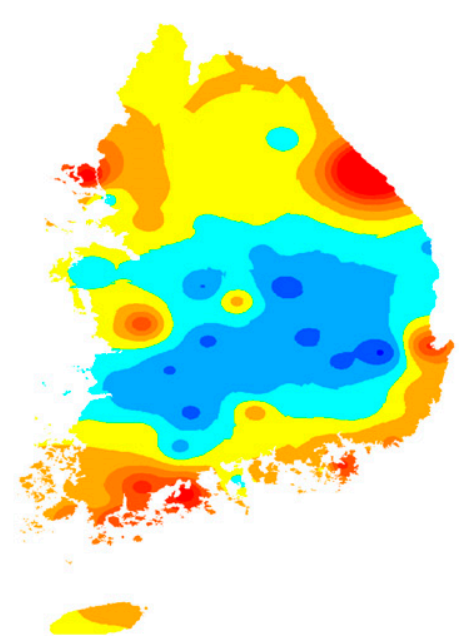

FIG. 12. As in Fig. 10, but for the 200-yr return period.

indicate a good performance in estimating HTM and HOM model parameters. Annual maximum rainfalls in South Korea are selected to verify the performance of the HTM model on a real world case study. Extreme rainfalls in South Korea result from two main sources: typhoons and convective storms (i.e., Changma). From the results of the current study, we conclude the following:

1) The MHML algorithm is a good approach for the estimation of the parameters of a heterogeneous mixture distribution. The MHML algorithm shows a good performance in estimating most parameters, except for the shape parameter, when the sample size is very small (i.e., lower than 50). The MHML algorithm shows an overall good performance, although it requires a relatively long record length to estimate the shape parameter of the density component in HTM.

2) In most cases, when the return period is high, the quantiles of mixture distributions are larger than the quantiles of nonmixture distributions. Sometimes, nonmixture distributions lead to the underestimation of quantiles corresponding to high-return periods.

3) HTM (i.e., G-EV1) demonstrates the best fit for all performance measures in all but a few stations in South Korea. While the nonmixture distribution (i.e., EV1 distribution) leads to a bad fit in some stations, mixture distributions consistently lead to a stable fit in most stations.

4) Quantiles estimated by mixture distributions in coastal areas are much larger than quantiles estimated by the nonmixture distribution. We suspect that these stations show intensive mixture characteristics because they are more directly affected by typhoons. Thus, mixture distributions represent a reasonable choice for extreme rainfall frequency analysis, especially in coastal areas.

The MHML algorithm demonstrates a good estimation performance even when record lengths are short (i.e., <50). The application of mixture distributions to 

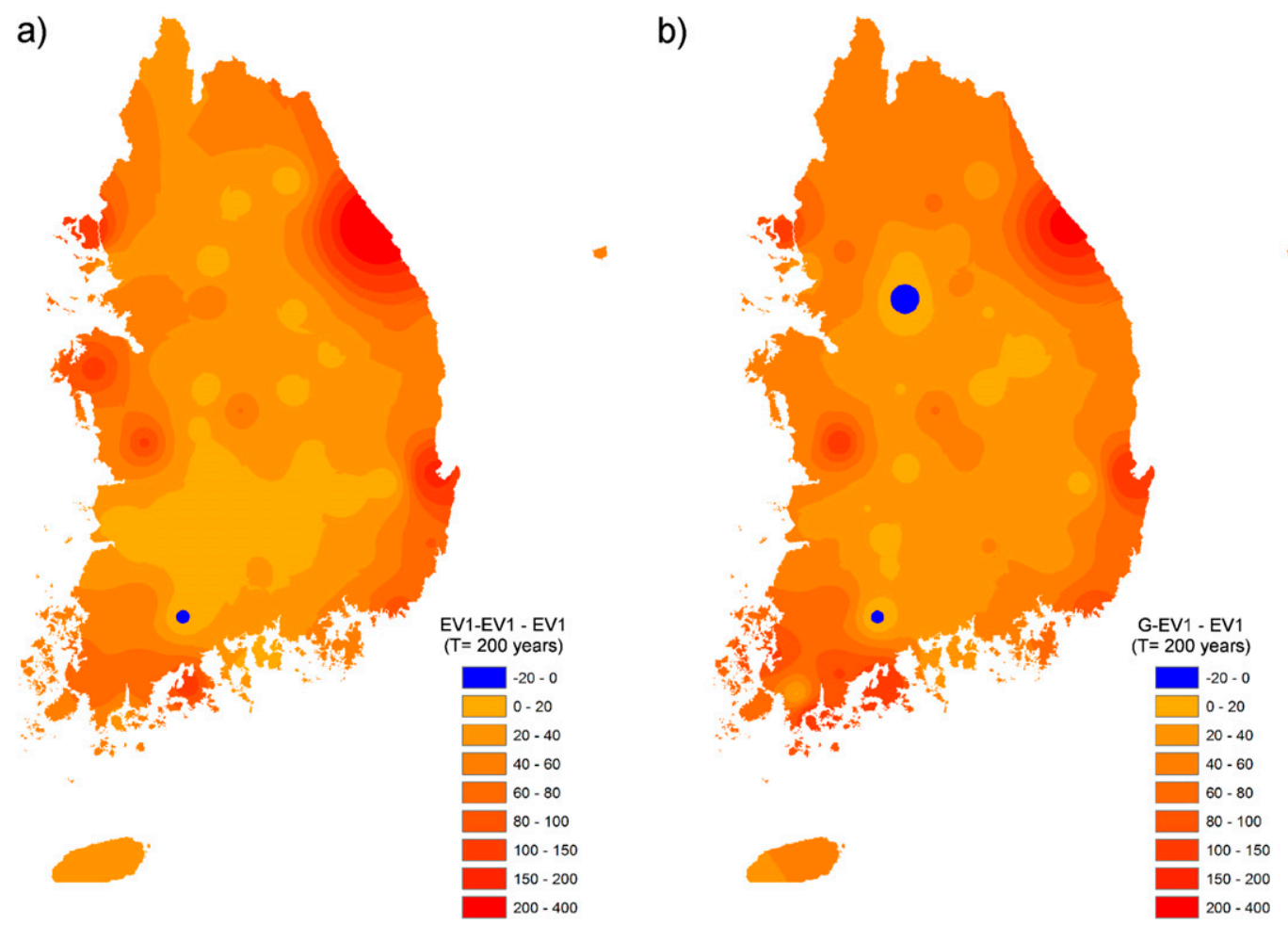

FIG. 13. As in Fig. 9, but for the 200-yr return period.

model various hydrometeorological variables and the development of methods for the selection of densities represent topics of interest for future research.

Acknowledgments. The authors thank the Korea Meteorological Administration for having provided the data used in this research work. The past observation database is available at http://www.kma.go.kr/weather/ climate/past_table.jsp. The authors would like also to thank the Chief Editor, Dr. Christa D. Peters-Lidard, and two anonymous reviewers for their comments and suggestions which helped improve the quality of the paper. The second author acknowledges that this research was supported by a grant ["Development of the Evaluation Technology for Complex Causes of Inundation Vulnerability and the Response Plans in Coastal Urban Areas for Adaptation to Climate Change" (MPSS-NH2015-77)] from the Natural Hazard Mitigation Research Group, Ministry of Public Safety and Security of Korea.

\section{APPENDIX}

\section{Genetic Algorithm}

Genetic algorithms (GAs) represent a way of solving optimization problems by mimicking the mechanics of natural selection and natural genetics. Critical terminology used in GAs is gene, individual, population, and generation. Gene means the information (i.e., parameters) of each individual. Individual is a group of genes and represents a candidate parameter set. Population is a group of individuals. Generation indicates the iteration number corresponding to population. Figure A1 presents

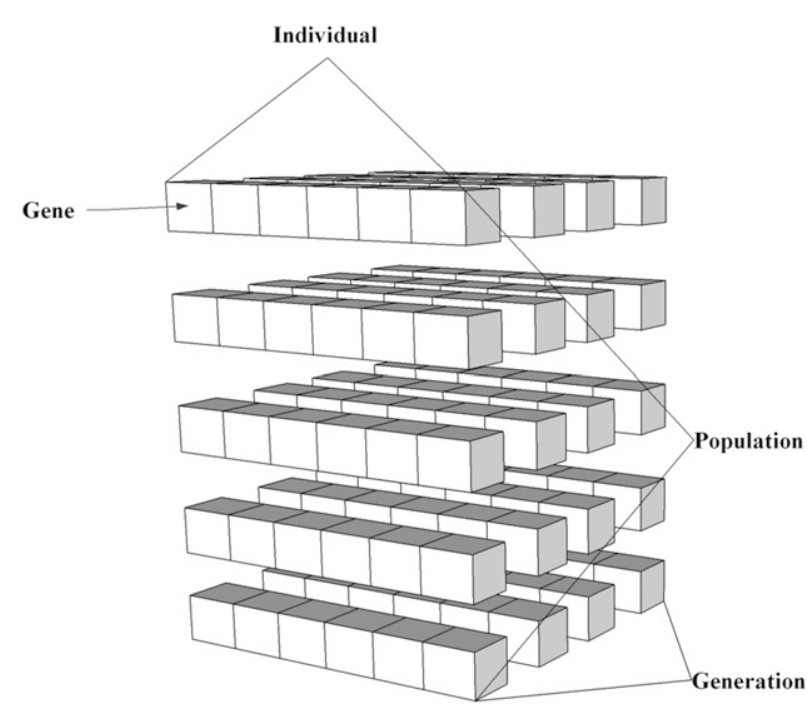

FIG. A1. Structure of the gene, the individual, the population, and the generation. 


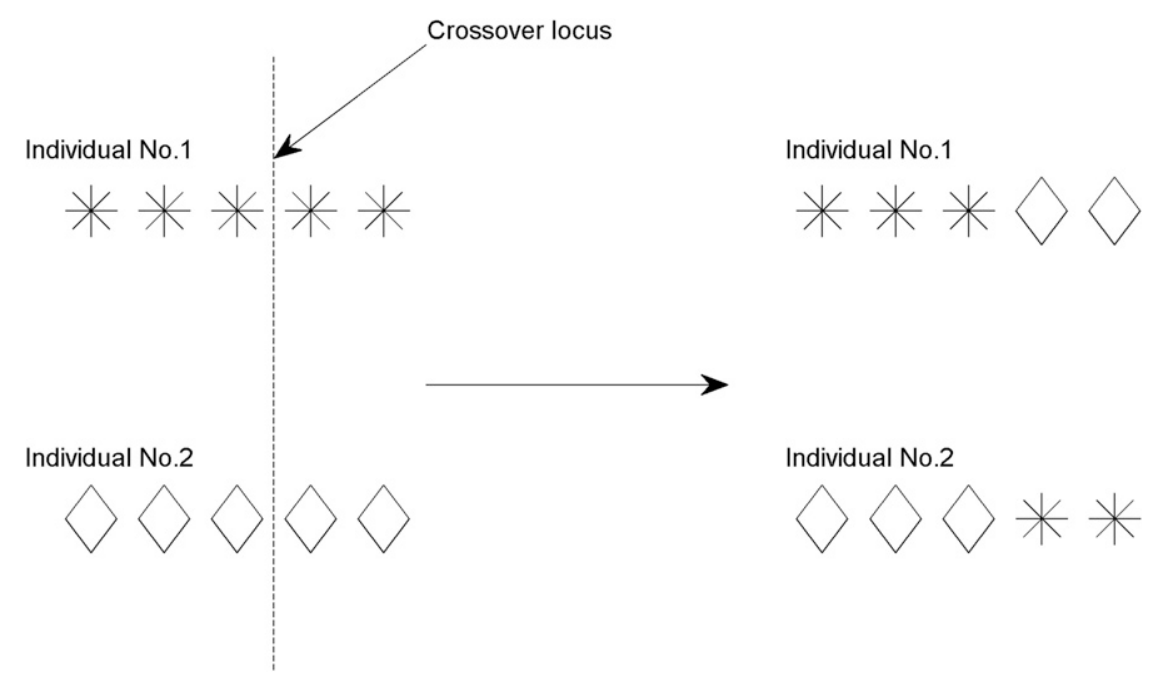

FIG. A2. Description of crossover operator.

the overall structure of GA including gene, individual, population, and generation.

GAs have three operators, namely, selection, crossover, and mutation. GAs start with an initial population that can be randomly generated. After generating an initial population, better individuals leading to a larger value of objective function are selected and passed on to the next generation. This work is done by the selection operator, and the selected individuals become parents. Crossover operator generates new individuals (offspring) by mixing genes of the parents in the current population. When mixing the genes of parents, the locus separating genes in individual to two parts is randomly selected. Furthermore, GA requires an additional operator to change the values of genes that are not in the selected individuals. The mutation operator has a role of changing values of genes in a new individual generated by the crossover operator. Figures A2 and A3 describe the crossover and mutation operators, respectively. The following algorithm presents a brief procedure for the GA.

1) Randomly generate population of parameter sets.

2) Calculate the fitness (i.e., the value of objective function) for each individual.

3) Repeat the following steps until all $n$ offspring have been created:

(i) Select a pair of parent individuals from the current population with the probability of selection being an increasing function of fitness.
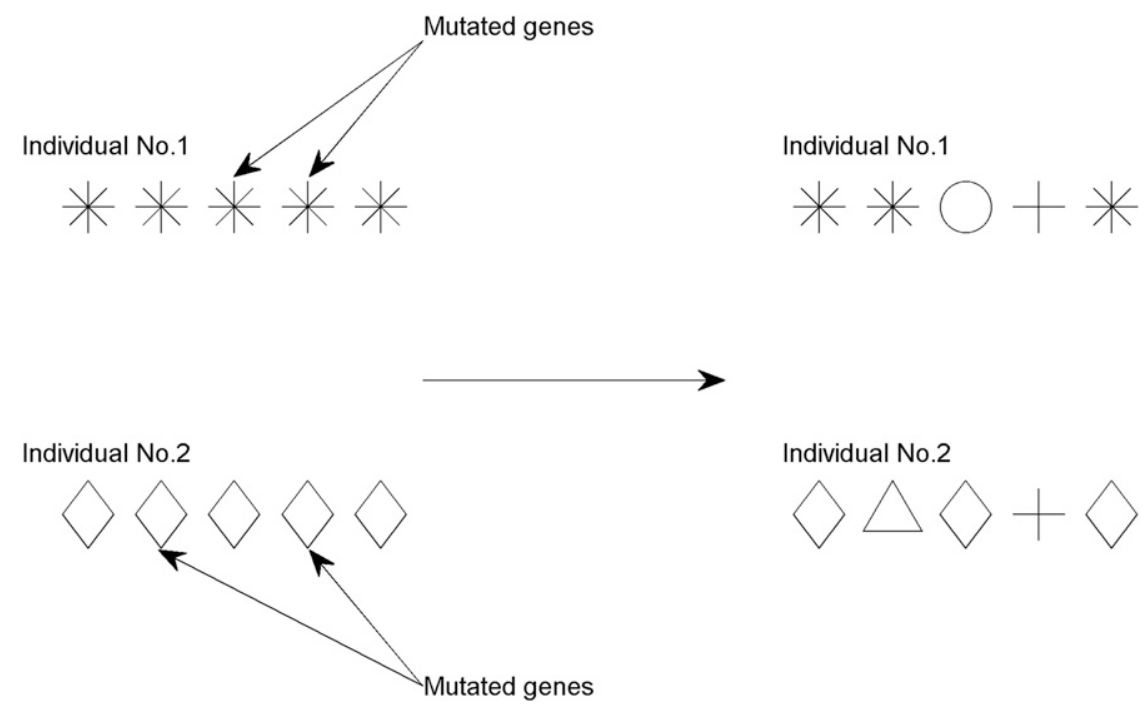

FIG. A3. Description of mutation operator. 
(ii) Cross over the selected pair at a randomly selected point to form two offspring with the crossover probability.

(iii) Mutate the two offspring at each locus with the mutation probability, and place the resulting individuals in the new population.

4) Replace the current population with the new population.

5) Go to step 2 until the fitness of the best individual reaches a given criteria or the number of the current generation meets the given maximum generation number.

More detailed information of the GA can be found in textbooks (Goldberg 1989; Mitchell 1998).

\section{REFERENCES}

Ahmad, M. I., C. D. Sinclair, and B. D. Spurr, 1988a: Assessment of flood frequency models using empirical distribution function statistics. Water Resour. Res., 24, 1323-1328, doi:10.1029/ WR024i008p01323.

—_, ——, and A. Werritty, 1988b: Log-logistic flood frequency analysis. J. Hydrol., 98, 205-224, doi:10.1016/ 0022-1694(88)90015-7.

Akaike, H., 1974: A new look at the statistical model identification. IEEE Trans. Autom. Control, 19, 716-723, doi:10.1109/ TAC.1974.1100705.

Alila, Y., and A. Mtiraoui, 2002: Implications of heterogeneous flood-frequency distributions on traditional stream-discharge prediction techniques. Hydrol. Processes, 16, 1065-1084, doi:10.1002/hyp.346.

Arnell, N. W., M. Beran, and J. R. M. Hosking, 1986: Unbiased plotting positions for the general extreme value distribution. J. Hydrol., 86, 59-69, doi:10.1016/0022-1694(86)90006-5.

Ashkar, F., and T. B. M. J. Ouarda, 1996: On some methods of fitting the generalized Pareto distribution. J. Hydrol., 177, 117-141, doi:10.1016/0022-1694(95)02793-9.

$\longrightarrow$, and -1998 : Approximate confidence intervals for quantiles of gamma and generalized gamma distributions. J. Hydrol. Eng., 3, 43-51, doi:10.1061/(ASCE)1084-0699(1998)3:1(43).

Calenda, G., C. P. Mancini, and E. Volpi, 2009: Selection of the probabilistic model of extreme floods: The case of the River Tiber in Rome. J. Hydrol., 371, 1-11, doi:10.1016/j.jhydrol.2009.03.010.

Cameron, D., K. Beven, and J. Tawn, 2000: Modelling extreme rainfalls using a modified random pulse Bartlett-Lewis stochastic rainfall model (with uncertainty). Adv. Water Resour., 24, 203-211, doi:10.1016/S0309-1708(00)00042-7.

Carreau, J., P. Naveau, and E. Sauquet, 2009: A statistical rainfallrunoff mixture model with heavy-tailed components. Water Resour. Res., 45, W10437, doi:10.1029/2009WR007880.

Carta, J. A., and P. Ramírez, 2007: Use of finite mixture distribution models in the analysis of wind energy in the Canarian Archipelago. Energy Convers. Manage., 48, 281-291, doi:10.1016/ j.enconman.2006.04.004.

Chebana, F., and T. B. M. J. Ouarda, 2008: Depth and homogeneity in regional flood frequency analysis. Water Resour. Res., 44, W11422, doi:10.1029/2007WR006771.

Chen, J., F. P. Brissette, and R. Leconte, 2010: A daily stochastic weather generator for preserving low-frequency of climate variability. J. Hydrol., 388, 480-490, doi:10.1016/ j.jhydrol.2010.05.032.

El-Adlouni, S., and T. B. M. J. Ouarda, 2009: Joint Bayesian model selection and parameter estimation of the generalized extreme value model with covariates using birth-death Markov chain Monte Carlo. Water Resour. Res., 45, W06403, doi:10.1029/ 2007WR006427.

,-- - X. Zhang, R. Roy, and B. Bobée, 2007: Generalized maximum likelihood estimators for the nonstationary generalized extreme value model. Water Resour. Res., 43, W03410, doi:10.1029/2005WR004545.

Erişoğlu, Ü., M. Erişoğlu, and H. Erol, 2011: A mixture model of two different distributions approach to the analysis of heterogeneous survival data. Int. J. Comput. Math. Sci., 5, 75-79.

Escalante-Sandoval, C., 1998: Multivariate extreme value distribution with mixed Gumbel marginals. J. Amer. Water Resour. Assoc., 34, 321-333, doi:10.1111/j.1752-1688.1998.tb04138.x.

Evin, G., J. Merleau, and L. Perreault, 2011: Two-component mixtures of normal, gamma, and Gumbel distributions for hydrological applications. Water Resour. Res., 47, W08525, doi:10.1029/2010WR010266.

Geem, Z. W., J. H. Kim, and G. V. Loganathan, 2001: A new heuristic optimization algorithm: Harmony search. Simulation, 76, 60-68, doi:10.1177/003754970107600201.

Goldberg, D. E., 1989: Genetic Algorithms in Search, Optimization, and Machine Learning. Addison-Wesley, $432 \mathrm{pp}$.

Hamed, K., and A. R. Rao, 2010: Flood Frequency Analysis. Taylor and Francis, $376 \mathrm{pp}$.

Hassanzadeh, Y., A. Abdi, S. Talatahari, and V. Singh, 2011: Metaheuristic algorithms for hydrologic frequency analysis. Water Resour. Manage., 25, 1855-1879, doi:10.1007/s11269-011-9778-1.

Heo, J. H., Y. W. Kho, H. Shin, S. Kim, and T. Kim, 2008: Regression equations of probability plot correlation coefficient test statistics from several probability distributions. J. Hydrol., 355, 1-15, doi:10.1016/j.jhydrol.2008.01.027.

Hu, Y.-M., Z.-M. Liang, Y.-W. Liu, X.-F. Zeng, and D. Wang, 2015: Uncertainty assessment of estimation of hydrological design values. Stochastic Environ. Res. Risk Assess., 29, 501-511, doi:10.1007/s00477-014-0979-z.

Hundecha, Y., A. St-Hilaire, T. B. M. J. Ouarda, S. El Adlouni, and P. Gachon, 2008: A nonstationary extreme value analysis for the assessment of changes in extreme annual wind speed over the Gulf of St. Lawrence, Canada. J. Appl. Meteor. Climatol., 47, 2745-2759, doi:10.1175/2008JAMC1665.1.

_ M. Pahlow, and A. Schumann, 2009: Modeling of daily precipitation at multiple locations using a mixture of distributions to characterize the extremes. Water Resour. Res., 45, W12412, doi:10.1029/2008WR007453.

Katz, R. W., 1999: Extreme value theory for precipitation: Sensitivity analysis for climate change. Adv. Water Resour., 23, 133139, doi:10.1016/S0309-1708(99)00017-2.

— , and X. Zheng, 1999: Mixture model for overdispersion of precipitation. J. Climate, 12, 2528-2537, doi:10.1175/ 1520-0442(1999)012<2528:MMFOOP > 2.0.CO;2.

_, M. B. Parlange, and P. Naveau, 2002: Statistics of extremes in hydrology. Adv. Water Resour., 25, 1287-1304, doi:10.1016/ S0309-1708(02)00056-8.

Kennedy, J., and R. Eberhart, 1995: Particle swarm optimization. Proc. Int. Conf. on Neural Networks, Vol. 4, Perth, WA, Australia, IEEE, 1942-1948, doi:10.1109/ICNN.1995.488968.

Kim, K., and Coauthors, 2000: Survey report of water resource management method development in 1999: Drawing Korean probability rainfall map. Korea Institute of Civil Engineering Tech. Rep. 
Kleiber, W., R. W. Katz, and B. Rajagopalan, 2012: Daily spatiotemporal precipitation simulation using latent and transformed Gaussian processes. Water Resour. Res., 48, W01523, doi:10.1029/2011WR011105.

Kwon, H. H., A. F. Khalil, and T. Siegfried, 2008: Analysis of extreme summer rainfall using climate teleconnections and typhoon characteristics in South Korea. J. Amer. Water Resour. Assoc., 44, 436-448, doi:10.1111/j.1752-1688.2008.00173.x.

Leclerc, M., and T. B. M. J. Ouarda, 2007: Non-stationary regional flood frequency analysis at ungauged sites. J. Hydrol., 343, 254-265, doi:10.1016/j.jhydrol.2007.06.021.

Lee, T., and T. B. M. J. Ouarda, 2010: Long-term prediction of precipitation and hydrologic extremes with nonstationary oscillation processes. J. Geophys. Res., 115, D13107, doi:10.1029/ 2009JD012801.

— tributed hydrometeorological extremes associated with largescale climate variability applied to South Korea. J. Appl. Meteor. Climatol., 53, 1193-1212, doi:10.1175/JAMC-D-13-0200.1.

Li, Z. Z., and Y. Zhang, 2011: Application of Gaussian mixture model and estimator to radar-based weather parameter estimations. IEEE Geosci. Remote Sens. Lett., 8, 1041-1045, doi:10.1109/ LGRS.2011.2151250.

Martins, E. S., and J. R. Stedinger, 2000: Generalized maximumlikelihood generalized extreme-value quantile estimators for hydrologic data. Water Resour. Res., 36, 737-744, doi:10.1029/ 1999WR900330.

Masina, M., and A. Lamberti, 2013: A nonstationary analysis for the northern Adriatic extreme sea levels. J. Geophys. Res. Oceans, 118, 3999-4016, doi:10.1002/jgrc.20313.

McLachlan, G. J., and D. Peel, 2000: Finite Mixture Models. Wiley, $456 \mathrm{pp}$.

Mitchell, M., 1998: An Introduction to Genetic Algorithms. MIT Press, $221 \mathrm{pp}$.

Nadarajah, S., and D. Choi, 2007: Maximum daily rainfall in South Korea. J. Earth Syst. Sci., 116, 311-320, doi:10.1007/ s12040-007-0028-0.

Ouarda, T. B. M. J., and C. Shu, 2009: Regional low-flow frequency analysis using single and ensemble artificial neural networks. Water Resour. Res., 45, W11428, doi:10.1029/2008WR007196. _ , and S. El-Adlouni, 2011: Bayesian nonstationary frequency analysis of hydrological variables. J. Amer. Water Resour. Assoc., 47, 496-505, doi:10.1111/j.1752-1688.2011.00544.x.

Overeem, A., A. Buishand, and I. Holleman, 2008: Rainfall depthduration-frequency curves and their uncertainties. J. Hydrol., 348, 124-134, doi:10.1016/j.jhydrol.2007.09.044.

Park, J.-S., H.-S. Kang, Y. S. Lee, and M.-K. Kim, 2011: Changes in the extreme daily rainfall in South Korea. Int. J. Climatol., 31, 2290-2299, doi:10.1002/joc. 2236

Phien, H. N., and T. Jivajirajah, 1984: Fitting annual rainfall an annual streamflow by two transformed Gamma distributions. Water $S A, \mathbf{1 0}, 65-74$.

Rossi, F., M. Fiorentino, and P. Versace, 1984: Two-component extreme value distribution for flood frequency analysis. Water Resour. Res., 20, 847-856, doi:10.1029/WR020i007p00847.

Russo, S., A. Dosio, A. Sterl, P. Barbosa, and J. Vogt, 2013: Projection of occurrence of extreme dry-wet years and seasons in Europe with stationary and nonstationary Standardized Precipitation Indices. J. Geophys. Res. Atmos., 118, 7628-7639, doi:10.1002/jgrd.50571.

Schaefli, B., D. B. Talamba, and A. Musy, 2007: Quantifying hydrological modeling errors through a mixture of normal distributions. J. Hydrol., 332, 303-315, doi:10.1016/j.jhydrol.2006.07.005.
Shin, J.-Y., J.-H. Heo, C. Jeong, and T. Lee, 2014: Meta-heuristic maximum likelihood parameter estimation of the mixture normal distribution for hydro-meteorological variables. Stochastic Environ. Res. Risk Assess., 28, 347-358, doi:10.1007/ s00477-013-0753-7.

Singh, V. P., S. Wang, and L. Zhang, 2005: Frequency analysis of nonidentically distributed hydrologic flood data. J. Hydrol., 307, 175-195, doi:10.1016/j.jhydrol.2004.10.029.

Smith, J. A., G. Villarini, and M. L. Baeck, 2011: Mixture distributions and the hydroclimatology of extreme rainfall and flooding in the eastern United States. J. Hydrometeor., 12, 294-309, doi:10.1175/2010JHM1242.1.

Strupczewski, W. G., K. Kochanek, E. Bogdanowicz, and I. Markiewicz, 2012: On seasonal approach to flood frequency modelling. Part I: Two-component distribution revisited. $H y$ drol. Processes, 26, 705-716, doi:10.1002/hyp.8179.

Sun, A. Y., A. P. Morris, and S. Mohanty, 2009: Sequential updating of multimodal hydrogeologic parameter fields using localization and clustering techniques. Water Resour. Res., 45, W07424, doi:10.1029/2008WR007443.

Tramblay, Y., A. St-Hilaire, and T. B. M. J. Ouarda, 2008: Frequency analysis of maximum annual suspended sediment concentrations in North America. Hydrol. Sci. J., 53, 236-252, doi:10.1623/hysj.53.1.236.

Vogel, R. M., 1986: Probability plot correlation coefficient test for the normal, lognormal and Gumbel distributional hypotheses. Water Resour. Res., 22, 587-590, doi:10.1029/WR022i004p00587.

Vrac, M., and P. Naveau, 2007: Stochastic downscaling of precipitation: From dry events to heavy rainfalls. Water Resour. Res., 43, W07402, doi:10.1029/2006WR005308; Corrigendum, 44, W05702, doi:10.1029/2008WR007083.

Waylen, P., and M.-K. Woo, 1983: Annual floods in southwestern British Columbia, Canada. J. Hydrol., 62, 95-105, doi:10.1016/ 0022-1694(83)90096-3.

Wilks, D. S., and R. L. Wilby, 1999: The weather generation game: A review of stochastic weather models. Prog. Phys. Geogr., 23, 329-357, doi:10.1177/030913339902300302.

Wójcik, R., P. A. Troch, H. Stricker, P. Torfs, E. Wood, H. Su, and Z. Su, 2006: Mixtures of Gaussians for uncertainty description in bivariate latent heat flux proxies. J. Hydrometeor., 7, 330345, doi:10.1175/JHM491.1.

Yoo, C., K. S. Jung, and T. W. Kim, 2005: Rainfall frequency analysis using a mixed Gamma distribution: Evaluation of the global warming effect on daily rainfall. Hydrol. Processes, 19, 3851-3861, doi:10.1002/hyp.5985.

Yoon, P., T.-W. Kim, J.-S. Yang, and S.-O. Lee, 2012: Estimating quantiles of extreme rainfall using a mixed Gumbel distribution model. J. Korea Water Resour. Assoc., 45, 263-274, doi:10.3741/JKWRA.2012.45.3.263

_ — _ , and C. Yoo, 2013: Rainfall frequency analysis using a mixed GEV distribution: A case study for annual maximum rainfalls in South Korea. Stochastic Environ. Res. Risk Assess., 27, 1143-1153, doi:10.1007/s00477-012-0650-5.

Yoon, S., C. Jeong, and T. Lee, 2013: Application of harmony search to design storm estimation from probability distribution models. J. Appl. Math., 2013, 932943, doi:10.1155/ 2013/932943.

Yue, S., T. B. M. J. Ouarda, B. Bobée, P. Legendre, and P. Bruneau, 1999: The Gumbel mixed model for flood frequency analysis. J. Hydrol., 226, 88-100, doi:10.1016/S0022-1694(99)00168-7.

,-- , and -2001 : A review of bivariate gamma distributions for hydrological application. J. Hydrol., 246, 1-18, doi:10.1016/S0022-1694(01)00374-2. 